Review Article

\title{
Mission Planning Issues of Imaging Satellites: Summary, Discussion, and Prospects
}

\author{
Guohui Zhang $\mathbb{D}^{D}$, Xinhong Li, Gangxuan Hu $\mathbb{D}^{D}$, Zhibin Zhang $\mathbb{D}$, Jiping An $\mathbb{D}^{D}$, \\ and Wanxin Man
}

Department of Aerospace Science and Technology, Space Engineering University, Beijing 101416, China

Correspondence should be addressed to Guohui Zhang; 550894804@qq.com

Received 9 September 2021; Accepted 3 December 2021; Published 29 December 2021

Academic Editor: Giovanni Palmerini

Copyright (C) 2021 Guohui Zhang et al. This is an open access article distributed under the Creative Commons Attribution License, which permits unrestricted use, distribution, and reproduction in any medium, provided the original work is properly cited.

Satellite mission planning is the basis and top-level work of space missions and the beginning of each space mission. Therefore, the scientific research of satellite mission planning is very important. By analyzing the existing research results, we can know that the research on task planning mainly focuses on three aspects: research objects, established model, and solution algorithm. Starting from these three aspects vertically and then horizontally, this paper comprehensively discusses the theoretical basis, application, and advantages and disadvantages of related technologies in the research literature in recent years. Finally, based on the research on satellite mission planning, this paper puts forward its own views on the future development direction and research focus.

\section{Introduction}

The vast universe has been a mystery field that mankind has longed for since ancient times. Mankind's activities in exploring, researching, and using space are increasing day by day. Among them, remote sensing imaging is an important space activity that utilizes space resources. Remote sensing is a long-distance, noncontact target detection technology and method. It uses artificial satellites or other aircraft to detect targets, acquire target information, and then process the acquired information, thereby qualitatively positioning or quantitatively describing the target [1]. Remote sensing imaging can be used in many aspects such as meteorological observation, resource survey, map surveying and mapping, and military reconnaissance.

The effective execution of remote sensing missions by imaging satellites requires reasonable mission planning, which refers to a technique for optimizing the execution of satellite observation missions by reasonably allocating satellite system resources and formulating action sequences according to user requirements. Satellite mission planning plays a key role in the whole process of earth observation and therefore has been thoroughly studied by domestic and foreign scholars for a long time.

Solving the task planning problem can be divided into four phases: task details, model building, algorithm solution, and evaluation and optimization, as shown in Figure 1.

Scholars have also based their studies on these four aspects. In terms of task details, we focus on the classification of task planning problems, the decomposition of tasks, and the formal description of tasks. In the model building phase, the study focuses on how to model the task planning problem so that it can be solved with simulation algorithms. The existing task planning models can be broadly classified into three categories: planning models, constraint satisfaction models, and other models. In the algorithm solving stage, the main research is on the best algorithm that can solve the model to obtain the task planning solution. The reasonable choice of algorithm can make the problem solving fast and the solving result accurate. The algorithms used in the current study can be divided into deterministic algorithms, heuristic algorithms, and search algorithms. In recent years, the discipline of artificial intelligence has developed rapidly, and the research of solving task planning has 


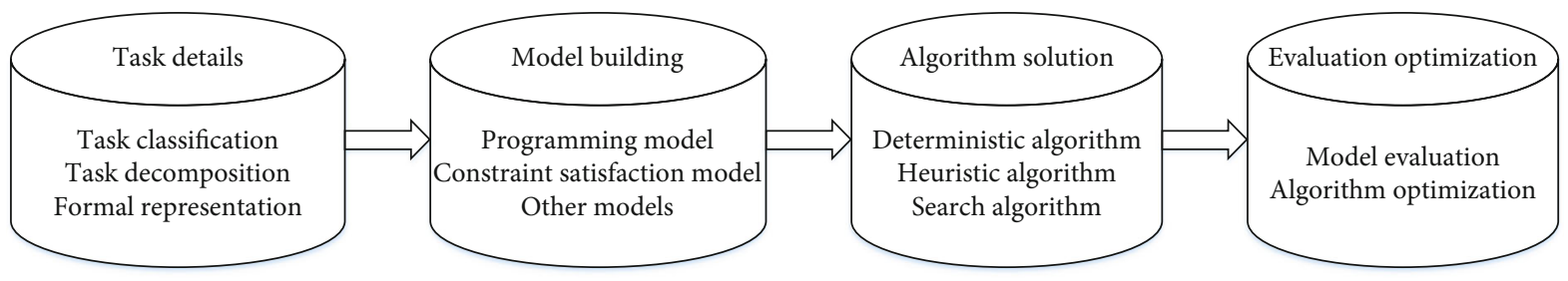

FIGURE 1: Flow chart of the four phases of mission planning.

emerged using artificial intelligence methods such as reinforcement learning and neural network algorithms. The final evaluation and optimization phase focuses on evaluating the rationality of the model and optimizing the algorithm.

Among them, model building and algorithm solution research are the key parts of the whole task planning research, and therefore, most of the scholars' research is focused on them. In this paper, 500 research papers from the last 15 years were selected and relevant data analysis was conducted using the literature analysis software SATI [2], as shown in Figures 2 and 3.

We analyzed the year of publication of the related literature, and it can be seen from Figure 2 that the research on satellite mission planning has been zigzagging along and the research fervor shows no signs of decay. Therefore, the review based on mission planning in this paper has some guidance for future research. Figure 3 shows the frequency analysis of the keywords in the selected 500 papers. After eliminating the most important "satellite mission planning" and other noisy keywords, we can see that the keywords can be divided into research object, model, algorithm, and others. In this paper, the three important directions of research objects, models, and algorithms are used as an overview idea. In Section 2, the objects of study are classified according to different classification principles, and the principles of the research problem are described separately. In Section 3, the commonly used models are elaborated. In Section 4, the algorithms are classified according to the different principles of optimization, and the characteristics, advantages, and disadvantages of each type of algorithm are focused on. The research ideas of task planning are clarified, the advantages of previous research are studied, some shortcomings of existing research are presented, and finally, some prospects are proposed for future scholars to make reference and study.

\section{Classification of Research Objects}

The mission planning problem has been studied in an extremely wide range and classified in a complicated way. This section provides an overview of the classification of the mission planning problem for earth observation satellites, using an example of an earth observation satellite. The composition and workflow of an earth observation satellite system are shown in Figure 4.

As can be seen in Figure 4, the main elements in the earth observation satellite system consist of satellites, ground stations, observation targets, and the environment. Classifying the satellite mission planning according to different

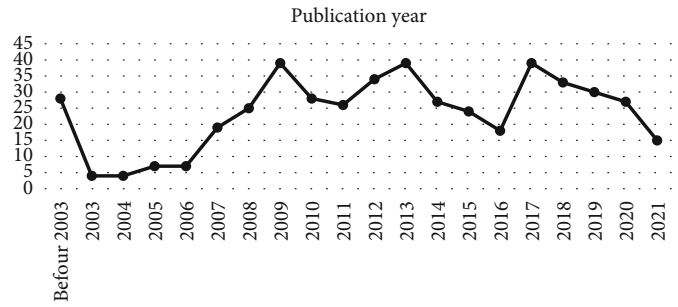

FIgURE 2: Overview of number of selected articles in years.

elements, there can be three different types of mission planning [3].

2.1. Classification by Number of Satellites and Payloads. Based on the number of satellites and payloads involved in mission planning scheduling, it can be divided into single-satellite single-payload mission planning, multisatellite single-payload planning, and multisatellite multipayload planning.

2.1.1. Single-Satellite Single-Payload Mission Planning. The single-satellite single-load mission planning problem is usually studied for one spacecraft with one optical lens on board. The problem has fewer constraints, clear optimization objectives, and relatively simple models, which can be abstracted as a rational sequencing problem for multiple observation tasks of one resource. The research on singlesatellite single-load mission planning started earlier, but due to the weak single-satellite reconnaissance capability and increasing reconnaissance demand, the research has progressed slowly in recent years, and most of it has been used as the basis for transitional research on multisatellite cooperative mission planning.

As early as 1994, Hall and Magazine [4] considered the visible time window constraint, modeled the space mission planning problem as a machine scheduling problem, developed a constraint satisfaction model, and applied various heuristic algorithms such as a Lagrangian relaxation algorithm to solve the model problem.

In 1996, Bensana et al. [5] developed the integer planning model for the first time for the single-satellite planning problem of SPOT-5 considering the platform storage capacity constraint, followed by Vasquez et al. [6] who then developed a constraint satisfaction model for the singlesatellite planning problem of SPOT-5 considering the platform storage capacity constraint and solved the model using a forbidden search algorithm. 


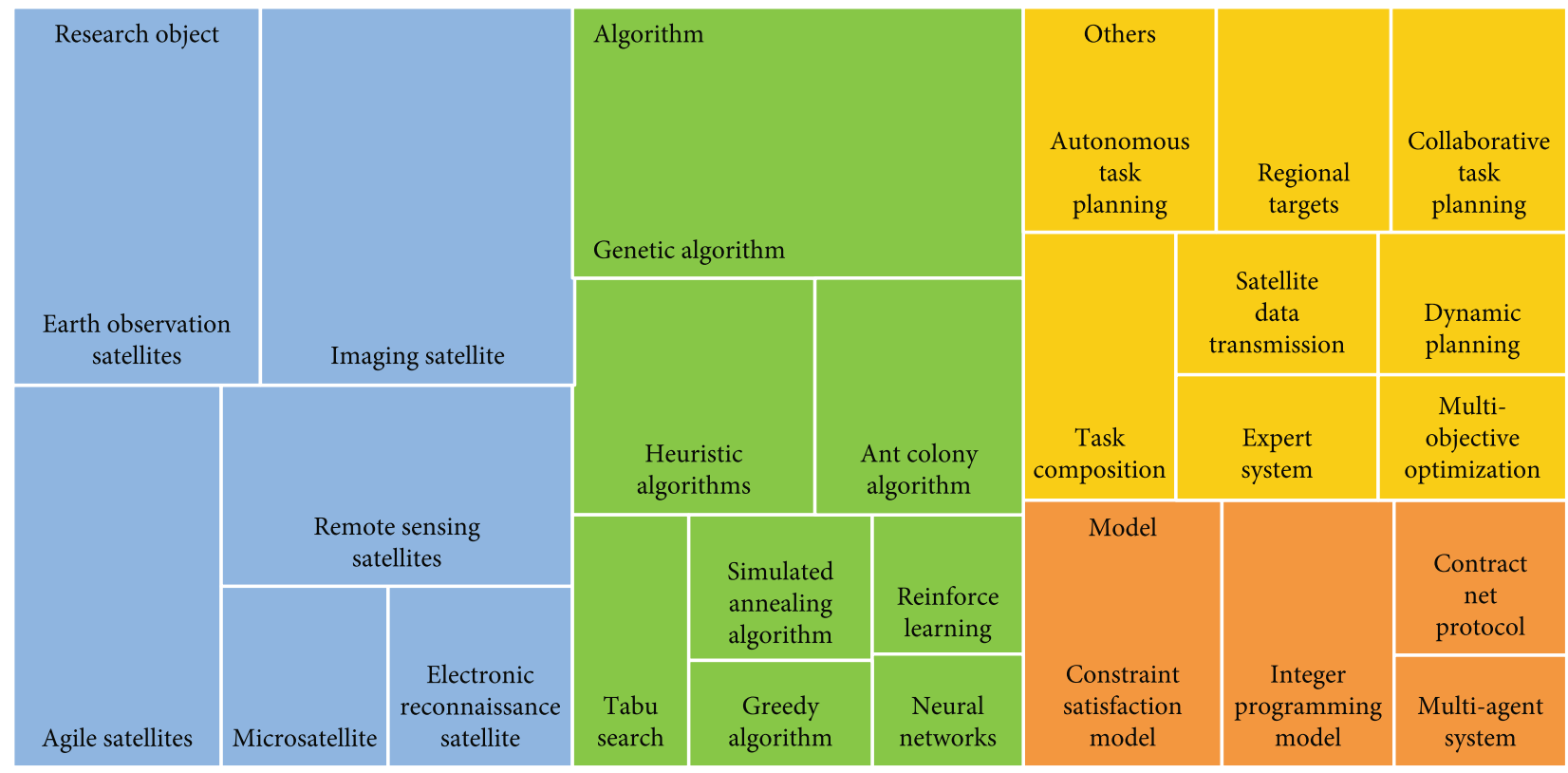

Key words

Research object
Model

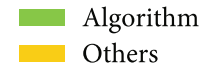

Figure 3: Article keyword frequency chart.

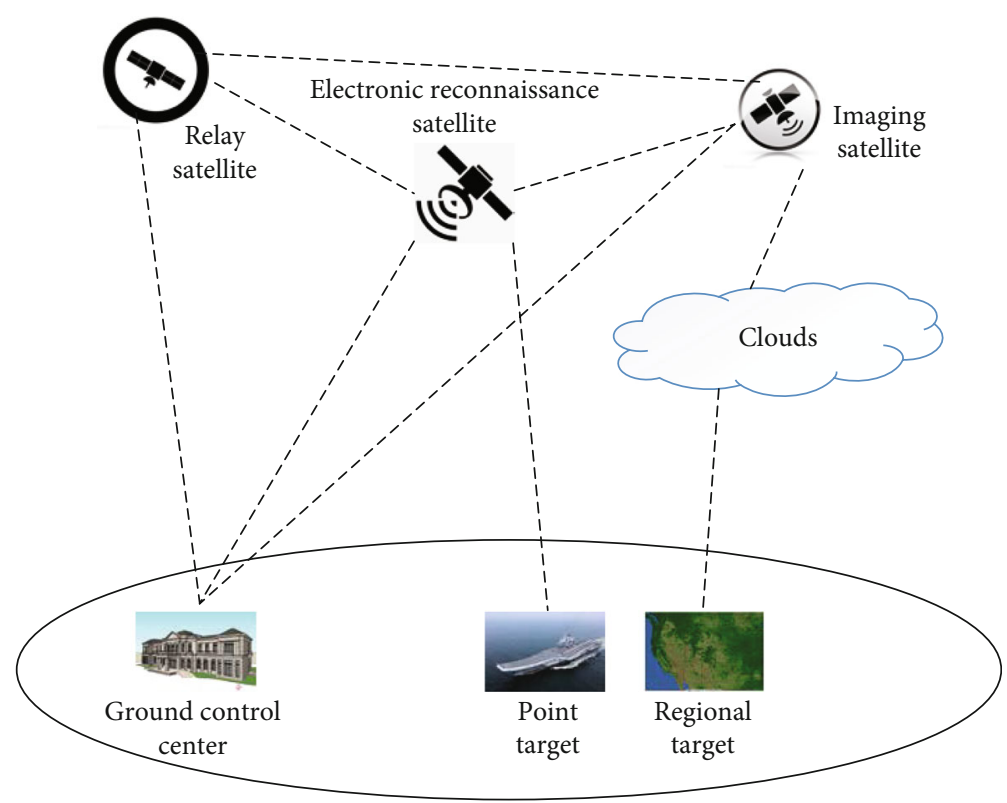

FIgURE 4: Diagram of earth observation satellite system.

In 2000, Wolfe and Sorensen [7] proposed three algorithms to solve the single-satellite single-orbit mission assignment problem for agile satellites: a priority-based mission assignment method, a forward-looking function with task assignment method, and a genetic algorithm with additional parameters based on these two algorithms.

After entering the 21 st century, the research on singlesatellite mission planning has become more in-depth. For the South Korean COMS satellite, Lee et al. [8] proposed that the task scheduling problem can be considered a specific form of the general job-shop scheduling problem, which requires the optimization of resource usage, and he proposed two algorithms, namely, First-Finished First-Scheduled Algorithm and Accommodating Discarded Task Algorithm.

In 2017, Peng et al. [9] present an approach to deal with the single satellite observation and transmission task 
planning problem. To solve this problem, they present a directed acyclic graph model and map the problem into an optimal path search problem.

In recent years, Chu et al. [10] regard the single-satellite planning problem as the satellite selecting a subset of targets that can meet all the observation constraints from the set of candidate observation targets for observation and obtaining the maximum observation benefit. Aiming at the timing constraints of satellites, they proposed a branch and bound algorithm that can solve the optimal solution of the singlesatellite single-orbit imaging problem. Yuan [11] designed a heuristic task loading algorithm based on the idea of online boxing for the task planning link at the single-satellite level, taking into account the scheduled tasks for the online task planning problem. The loading algorithm includes three types: direct loading, replacement loading, and replacement loading.

In the same period, Du et al. [12] introduced task clustering strategy when studying single satellite to point target imaging mission planning, which increased the observation efficiency and overcome the shortcomings of static task clustering strategy. When considering the study of target planning for a single star area with the constraint of drift angle, the problem is expressed as a traveling salesman problem (TSP) node planning problem through the grid discretization method, and an improve sense and consciousness ant colony optimization algorithm with branching and cutting strategies is developed to solve the constrained TSP node planning problem.

In 2020, Miao et al. [13] aimed at the observation of multiple targets on the ground by a single satellite, and based on the mission planning model and target level, the mission planning problem was transformed into an optimization problem with imaging revenue as the goal. Aiming at this optimization problem, she proposed a step-by-step allocation of on-orbit task planning algorithm and, on this basis, proposed a step-by-step on-orbit task planning algorithm that joins the best selection process.

In summary, the research on single-satellite mission planning mostly considers the satisfaction or optimization of single or partial simple constraints, and the established model is relatively simple. In the future, the research on single-satellites needs to consider actual constraints. As satellite applications become more and more widespread, user requirements are becoming more and more complex, and it is also necessary to study single-satellite autonomous intelligent task planning and intelligently handle emergency tasks.

2.1.2. Multisatellite Single-Load Mission Planning. The capacity of a satellite is limited. In order to improve the efficiency of observation, multiple satellites work together to form a distributed satellite system to jointly complete the observation task. With the continuous increase in the number of satellites, the continuous enhancement of satellite capabilities, and the continuous improvement of user demand complexity and timeliness, satellite mission planning has also moved from single-satellite self-planning to multiconstellation coordinated planning. Multisatellite scheduling and planning problems are more complicated, with more constraints and coupling between constraints. Due to the complexity of the task, multisatellite task planning has been proven to be a type of multiconstrained time-related NP-hard problem [14]. Most of the research on this problem focuses on the modeling of planning problems and the solution of different algorithms. According to different modeling purposes and research focuses, the hypothetical constraints and optimization objective functions are not the same.

As early as 2008, Wang et al. [15] compared the multisatellite mission planning problem with the 0-1 knapsack problem, took the imaging mission as an "item," reduced the reconnaissance time window to a "knapsack" with multiple attributes, and proposed a "compatibility list" to describe the complex correspondence between items and backpacks; a multidimensional dynamic backpack model was established.

According to the requirements of agile satellite missions, Hao et al. [16] constructed a multiobjective-based agile satellite mission planning mathematical model according to the characteristics of multiagile satellite networking mission planning.

In 2015, Nan [17] analyzed the effect of multisatellite joint observation in his doctoral dissertation and designed a multisatellite distributed mission planning system based on improved contract network protocol, including planning architecture and planning process. And the task planning system has been tested and verified experimentally.

In view of the characteristics of the multisatellite autonomous mission planning problem, Yingen [18] disassembled the multisatellite autonomous mission planning problem into two problems: multisatellite task allocation and singlesatellite autonomous mission planning. He combined satellite system modeling and satellite autonomous mission planning modeling, and based on the modeling ideas of constraint satisfaction models, he constructed a general model for multisatellite mission planning.

The research ideas for multisatellite and single-load mission planning are basically the same: that is, sort out the constraints of multisatellite mission planning, establish appropriate planning models, and develop optimal algorithms to solve them. The difference is the types of models and algorithms, which will be discussed in the next two chapters, so I will not repeat it here.

2.1.3. Multisatellite Multiload Mission Planning. For the convenience of modeling, most of the existing studies assume that each satellite carries only one load, and there are no cooperative and mutually exclusive constraints between satellites and loads. In fact, the development of modern science and technology has enabled satellites to carry multiple loads at the same time, and different loads can work at the same time or switch, and the working mode has become complex. This increases the difficulty of task planning, so the research on multiload task planning is rare.

As early as 2009, Yu-hua et al. [19] highly abstracted the multitype satellite loads, considering the working mode constraints and on-off time constraints of visible light, SAR and other loads. They regarded each possible switch of satellite 

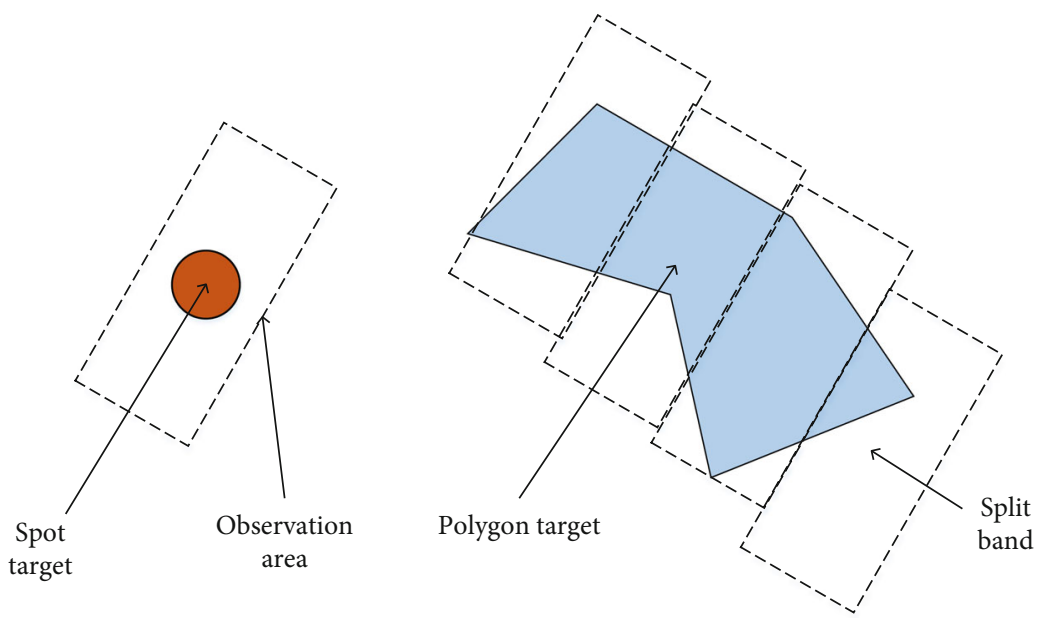

FIGURE 5: Schematic diagram of spot target and polygon target.

load as a working mode, established a unified load action mode, and then used the constraint description model for planning and description. However, the problem of a satellite carrying different types of loads was not considered in the planning process. Yang [20] considered the constraints of different load switching times, but in the actual modeling process, each satellite still carries only one sensor.

In 2011, Pan et al. [21] divided the multisatellite and multiload joint scheduling problem into two stages, namely, observation scheduling stage and data transmission scheduling stage, gave the optimization objectives and constraints of each stage, respectively, and established the multisatellite and multiload joint scheduling model based on stage optimization. Hao [22] introduced the multiagent system for the new multiload electromagnetic exploration satellite, established the multiload electromagnetic exploration satellite task driven planning model, and designed a multilevel hybrid collaborative planning algorithm based on multiagent, which effectively solved the multiload satellite task planning problem. In the same period, Yin [23] analyzed the working principle and characteristics of multiload satellite earth observation and considered the combination of multiple loads. The framework model of multiload earth observation satellite mission planning is established, and the target access calculation method is proposed.

Aiming at the problem of multiairship and multiload earth observation task planning, Longmei [24] used the ontology based modeling method to model the airship platform, load, and task; proposed the multiairship task allocation method based on discrete particle swarm optimization; and finally designed and implemented the multiairship and multiload earth observation task planning experimental system. Although his research is the research of near space vehicle mission planning, it also plays a guiding role in the research of multipayload satellite mission planning.

The classification of mission planning according to the number of satellites and loads is actually in line with the development law of research, from shallow to deep, from basic to complex, and from theory to practice. Single satellite mission planning is the simplified basis of all planning, and multisatellite and multiload mission planning is closely related to practice. Future research directions will focus on considering more and more practical constraints, improving accuracy and reducing simplified practical models.

2.2. Classification by the Type of Observation Target. In satellite earth observation, according to the different characteristics of observation targets, observation targets can be divided into spot targets and polygon targets. As shown in Figure 5, the spot targets include warships, aircraft carriers, launch bases, and other targets with a radius of less than $10 \mathrm{~km}$. They are widely distributed, numerous, and dense and have high military and civil value. The coverage observation of spot targets can be realized by a single satellite in one transit. Polygon targets include large cities, sea areas, islands, and other targets with a radius greater than the field of view of a single satellite imaging photo. They have a large area and require satellite sideways or even joint observation of multiple satellites to achieve coverage observation.

2.2.1. Mission Planning for Spot Targets. The spot target can be observed by a single satellite. Therefore, the spot targetoriented imaging satellite scheduling is essentially to arrange $N$ spot target observation tasks in $M$ observation equipment and determine the specific time of observation. The spot target oriented imaging satellite scheduling is relatively simple, which can be mapped to workshop scheduling, multidimensional knapsack, and other problems. By establishing planning models, constraint satisfaction problems, and other models, various optimization algorithms are used to solve them.

Geng et al. [25] proposed a cluster division algorithm based on vertex degree to solve the cluster problem of dense point observation tasks and form a series of cluster observation targets. In order to obtain the optimal mission target observation sequence, considering the target visible time window constraints and satellite agile mobility constraints, a performance index based on multitarget observation revenue and attitude maneuver energy consumption is 
constructed to realize energy-efficient mission planning. Finally, a fast observation task clustering strategy and heuristic ant colony optimization algorithm are proposed.

The research on spot target planning and the discussion on single satellite and multisatellite mission planning in the previous paper overlap and repeat more, so I will not repeat it here.

\subsubsection{Mission Planning for Polygon Objectives. For imaging} observation of polygon targets, joint observation by multiple satellites is required, as shown in Figure 6. For the research of task planning, it is generally divided into two subproblems: regional target decomposition and subtarget imaging task planning. It is quite different from task planning for spot targets. However, in fact, the observation task requirements submitted by users often include both spot objectives and polygon objectives. Therefore, it is necessary to comprehensively schedule the two objectives to complete the planning. However, in fact, due to the great difference in model description and algorithm design, most of the existing studies consider the two separately. A few studies first decompose the polygon target into spot target and then conduct comprehensive scheduling with spot target.

Regional target decomposition technology can be divided into static decomposition technology and dynamic decomposition technology. Static decomposition techniques mainly include single scene decomposition, predefined reference system decomposition, and strip decomposition with fixed width.

As early as 2006, Lemaître et al. [26] divides the irregular polygonal area target into parallel rectangular strips. When decomposing, the direction and offset of the strips need to be determined. For different strips, the width is equal, but the length is not fixed. Four quite different methods have been investigated in order to solve it: a fast greedy algorithm, a dynamic programming algorithm, a method based on the existing constraint programming framework, and a local search algorithm, based on insertion and removal of images in a sequence.

In 2008, Renjie et al. [27] proposed a dynamic decomposition method for polygonal targets, using satellite coverage of the area under different roll angles as the basis for decomposition, treating spot targets as special regional targets, and decomposing them according to observation opportunities. The metatasks after target decomposition are unified as the basic element of scheduling. Constructing income functions for different types of goals, respectively, reflects the difference in the calculation of the two types of goals and realizes the unified expression and processing of the two types of goals.

In 2015, Jing et al. [28] divided the area into multiple strips by mobile scanning with the satellite attitude with the camera field angle, so that the divided strips can completely cover the target area and ensure sufficient overlap width between adjacent strips. Nan [17] designed a "static and dynamic" polygon decomposition technology based on a longitude and latitude grid. Firstly, according to the static decomposition technology, the region to be split is decomposed into regular and manageable subregions and then

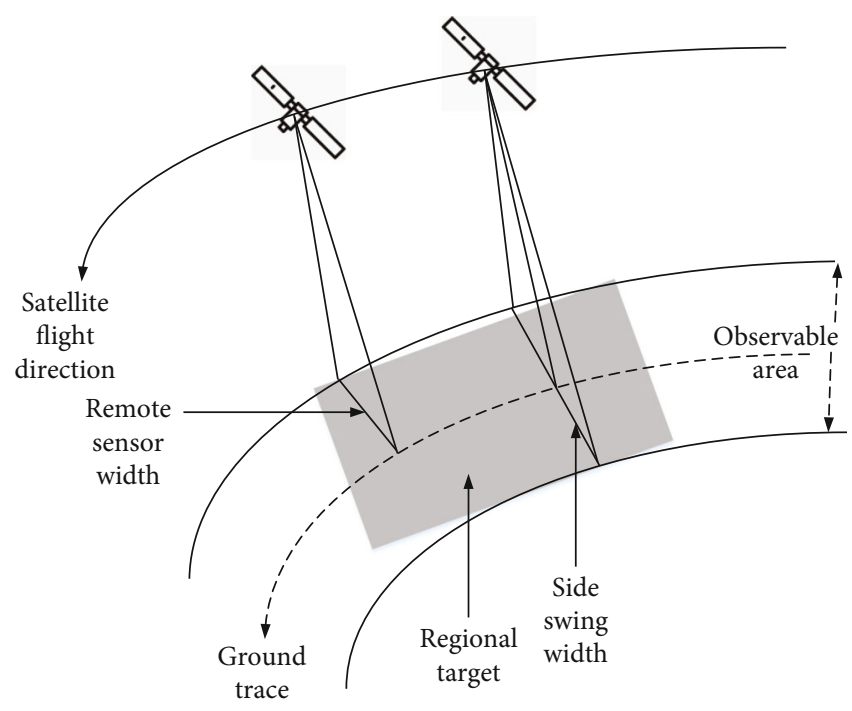

FIGURE 6: Joint observation of polygon targets.

dynamically combined according to the actual satellite orbit to merge into subregions conforming to the single observation of different satellites as an observation element task.

When studying the task of forest resources observation, Jianyin [29] transformed the forest observation area into a polygonal geometric target. Then, based on the characteristics of resolution, imaging type, and visual window of different load resources, the coverage area target segmentation algorithm and regional target decomposition process are proposed. The algorithm of regional target point lattice and adjacent point lattice unit combination is designed to obtain the metatask set, which provides task input for subsequent planning and scheduling.

The latest development is in 2020; Jingyu [30] improved the traditional polygonal target parallel segmentation method, enumerated all accessible observation modes under each observation opportunity of the satellite by using the side swing angle of the satellite, designed a Cell-based Dynamic Stripe Segmentation Method (CDSSM), and then established a multisatellite scheduling model for polygonal targets with unequal observation income. A heuristic algorithm based on weight priority strategy heuristic (WPSH) is designed, and a random neighborhood local search (RNLS) algorithm is proposed.

It can be seen that the existing research on polygonal goal task planning divides it into two parts: polygonal goal decomposition and task planning. In the problem of regional target decomposition, it is necessary to select an appropriate decomposition method according to different satellite orbits and observation targets.

In the future, the observation task planning of point targets and regional targets should also be deeply studied in the following aspects.

(1) Combined Observation Task Planning of Spot Target and Polygonal Target. The actual user observation needs are often the combination of point objectives and regional objectives. When planning both at the same time, the 
existing decomposition and planning method can not be well solved. In the future, we should study the joint planning of point objectives and regional objectives for collaborative observation.

(2) Multisatellite Cooperative Observation Task Planning for Targets in Different Regions. Practical satellite observation applications, such as forest resources survey and earthquake disaster relief, require multiple satellites to observe multiple regional targets. After increasing the number of satellites and targets, the requirements for planning are further increased.

\subsection{Classification according to Observed Working} Environment. According to the working environment of satellite observation mission, mission planning can be divided into static planning and dynamic planning. As the name suggests, static planning means that all environmental information is known before the satellite performs the mission, and the environment will not change during the satellite's mission. After the mission is planned on the ground, the command is uploaded to the satellite, and the satellite performs the observation mission according to the command. This is an idealized planning method, but in practice, the satellite operating environment can not be unchanged, and all environmental information can not be known in advance. Dynamic planning is the satellite mission planning to deal with the environmental changes under the condition of incomplete information.

The current mission planning research is mainly oriented to static planning, modeling, and algorithmic research on routine mission planning. This problem can be simply abstracted into a traveling salesman or path planning problem. The scholars' research on modeling and algorithms will be specifically elaborated in the following article.

Compared with static planning, dynamic planning is more difficult to study but more realistic and has guiding significance for realistic satellite observation mission planning.

As early as 1999, Schiex [31] once proposed an idea to solve the dynamic scheduling problem with new tasks arriving. Assuming that the task change in the original scheme caused by the insertion of new tasks is only to change the time of task arrangement and the task cannot be deleted from the original scheme, the new task can be inserted into the scheduling scheme.

Until 2002, Lemaître et al. [26] and others proposed an autonomous task scheduling algorithm based on sequential decision model in view of the dynamic change of cloud amount of imaging task of France Pleiades agile remote sensing satellite project. Cpa and Ggb [32] of Voretian company of France analyzed the requirements of multisatellite dynamic scheduling, divided the dynamic requirements of multisatellite into four types, including the state change of satellite resources, the arrival of new tasks, the selection of task opportunities, and the impact of environmental uncertainty, and pointed out the solution requirements with the smallest difference between the two successive schemes.

Based on the dynamic constraint satisfaction theory, Liu et al. [33] established a multisatellite dynamic scheduling model with new task arrival and gave the solution algorithm of the model in 2005.

In 2007, Wang et al. [34] considered the complex environmental impact in satellite work. Satellite observation task arrangement is faced with different uncertainties and disturbances, such as unforeseen cloud cover, unexpected changes in satellite resources, or the arrival of new tasks. Based on the detailed analysis of these dynamic factors, this paper uses a unified form to insert new tasks to describe the problem, proposes a rule-based heuristic algorithm, and designs a maximum contraction heuristic rule and a minimum occupancy heuristic rule for insertion.

In 2011, Grasset-bourdel et al. [35] studied the problem of off-line rescheduling using the ground scheduler when receiving an emergency observation request during the implementation of the plan and referred to the management agile satellite of MUSIS (Multinational space surveillance, reconnaissance and observation imaging system). The data included in the rescheduling includes not only the current activity plan but also some (up to dozens of) emergency observation requests. The goal is to quickly establish a new plan for the rest of the observation period.

In 2014, Wang et al. [36] proposed a heuristic algorithm based on two heuristic factors: time window congestion and task overlap to plan dynamic tasks of different sizes.

Chaochao [37] proposed a time window priority heuristic factor to ensure that the emergency task can be completed as soon as possible before the deadline in 2019. He designed a multicycle and multistage emergency scheduling strategy combining fixed cycle overall decision planning and local adjustment planning based on measurement and control station, optimized the scheduling time, and comprehensively solved the emergency task planning including conventional tasks.

In the same year, Yuan [11] has established the unified model of the first mock exam for multisatellites. Based on the online packing theory, a dynamic task loading algorithm is designed for the single satellite level task. A multisatellite online task planning solution framework based on the contract net protocol is built, and a variety of task assignment contracts are given. Bidding and bid evaluation links to adapt to the online task planning environment. Simulation examples show the effectiveness of the method in solving dynamic programming problems online.

From the above research status, scholars at home and abroad have carried out a series of dynamic programming research on dynamic tasks caused by dynamic factors such as the change of task demand and resource conditions. They mainly use optimization algorithm, intelligent optimization algorithm, and heuristic algorithm as the basic solution algorithm, and the solution efficiency of each algorithm is different. However, at present, the research on dynamic tasks mainly stays at the ground offline end and often only involves the single satellite level. Due to the limited observation capacity of single satellite, offline scheduling can not achieve rapid response to disturbance factors. Therefore, we need to comprehensively consider the previous research on multisatellite and multiload task planning and multiobjective joint task planning, so as to make the research more realistic and serve the reality. 
2.4. Summary of This Chapter. Satellite mission planning is a huge and complex system problem, which has been proved to be NP hard problem. In order to study it thoroughly, we must classify it and sort out the framework of mission planning. Starting with the object of mission planning, this section first introduces the composition of satellite observation system and then classifies it according to the constituent elements. Satellite mission planning is divided into single satellite single load mission planning, multisatellite single load mission planning, and multisatellite multiload mission planning according to satellites and loads. According to the observation target, it is divided into point target task planning and regional target task planning. According to the observation environment, it is divided into static task planning and dynamic task planning. It also expounds the research of scholars at home and abroad on the planning types under the classification and puts forward some suggestions for future research directions.

\section{Model Building}

In the process of solving task planning problem, the establishment of model is the first and most important link. The whole process of actual satellite system mission planning can be reproduced by using the model, and the actual mission planning can be designed and modified through the test of the model. In the simulation model, the all-physical model is the model type with the highest accuracy and can restore the real system, but its price is expensive, especially for the space environment; it is too difficult to realize. Therefore, most studies at home and abroad use mathematical model or physical mathematical model.

The establishment of the model varies from person to person, especially for the NP hard problem such as satellite mission planning; it is difficult to consider all constraints to establish a fine model. Different research focuses on the neglected elements which are different, so we need to take different methods to establish an appropriate model. This section will summarize the models established in satellite mission planning, which are divided into the following five types according to different methods, as shown in Figure 7. Then, it deeply analyzes the modeling ideas and application scope of each model and makes a comprehensive evaluation of each model. These provide guidance for a series of followup research, such as the reconstruction and optimization of task planning model, and have very important theoretical and practical significance.

3.1. Constraint Satisfaction Model. Constraint satisfaction problem (CSP) was first proposed by Huffman in 1971 and gave the formal definition of CSP [38]. A CSP is composed of a set of variables, the value range of each variable, and the constraints that limit the value of variables. It is usually used to solve the problems of scheduling optimization, trajectory optimization, path optimization, and so on. CSP is to solve these problems by finding an assignment for all variables within the value range of variables so that they meet all constraints.

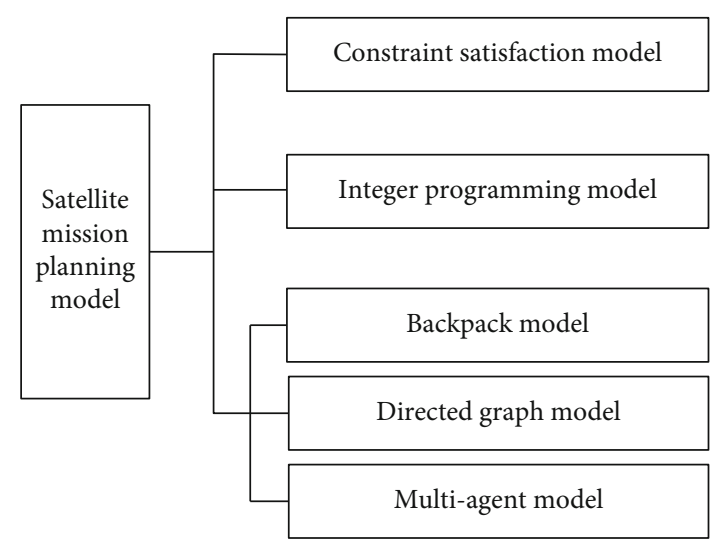

FIgURE 7: Classification of task planning models.

The core idea of satellite mission planning is to find a feasible observation action sequence on the premise of meeting these constraints according to the constraints such as mission observation requirements, satellite resource status, and execution task conflict. Therefore, the constraint satisfaction model can be well used to describe the satellite mission planning problem. Next, this paper will establish a basic constraint satisfaction model of satellite mission planning.

The model can be formally expressed as

$$
M=\langle T, S, A, C\rangle
$$

Among them, $T=\left\{T_{1}, T_{2}, \cdots, T_{n}\right\}$ is the task requirement set, $n$ represents the total number of tasks, and each task $T_{i}$ in the task set can be described as the following six tuples:

$$
\left\langle T N_{i}, T L_{i}, T P_{i}, T S_{i}, T E_{i}, T D_{i}\right\rangle
$$

Among them, $T N_{i}$ represents the number of task $T_{i}, T L_{i}$ represents the load type required by observation task $T_{i}, T P_{i}$ represents the observation income of task $T_{i}, T S_{i}$ represents the start time of observation of task $T_{i}, T E_{i}$ represents the end time of observation of task $T_{i}$, and $T D_{i}$ represents the continuous observation time required by task $T_{i}$.

$S=\left\{S_{1}, S_{2}, \cdots, S_{m}\right\}$ is the set of all satellites, where $m$ represents the total number of satellites. Each satellite can be described by the following six tuples:

$$
\left\langle S N_{i}, S S_{i}, S O_{i}, S L_{i}, S C_{i}, S P_{i}\right\rangle
$$

Among them, $S N_{i}$ represents the number of satellite $S_{i}$, $S S_{i}$ represents the load type carried by satellite $S_{i}, S O_{i}$ represents the observation resolution of satellite $S_{i}$ load, $S L_{i}$ represents the orbital position information of satellite $S_{i}$, $S C_{i}$ represents the on-board storage capacity function of satellite $S_{i}$, and $S P_{i}$ represents the energy and electricity function of satellite $S_{i}$.

$A$ and $C$ are the action set and constraint set taken by the satellite, which can be represented by multivariate arrays, respectively. 
Constraints are the focus of model establishment, and satellite mission planning is feasible only on the basis of meeting constraints. The actual constraints are various and complex, involving satellite operation, target observation, instruction annotation, result transmission, and so on. This section is the theoretical research on the constraint satisfaction model, so it is simplified. Only some constraints are considered as follows:

(1) Within any time interval $\Delta T$ when any satellite $S_{j}$ performs a task $T_{i}$, the memory occupied by tasks and other activities cannot exceed the total memory of the satellite

$$
\forall \Delta t: S C_{j}+T S C_{i}\left(\Delta t, S_{j}\right) \leq S C_{\operatorname{Max}}
$$

(2) Within any time interval $\Delta T$ for any satellite $S_{j}$ to perform a task $T_{i}$, the power consumption for performing tasks and other activities shall not exceed the total power consumption of the satellite

$$
\forall \Delta t: S P_{j}+T S P_{i}\left(\Delta t, S_{j}\right) \leq S P_{\mathrm{Max}}
$$

(3) The start time of the next task is greater than the sum of the end time of the current task and the satellite yaw maneuver time, wherein $t_{\text {sle }}$ is the yaw maneuver time after satellite $S_{j}$ completes task $T_{i}$

$$
T S_{i+1}>T E_{i}+t_{s l e}
$$

The optimization objective function is the result of model solving. The different emphasis of satellite mission observation requires different optimization objective functions to describe. Common optimization objective functions include the largest total task revenue, the largest saving of resources occupied by the task, and the priority of highpriority tasks. Taking the maximum total task revenue as an example, the optimization objective function $l(i)$ is as follows:

$$
l(i)=\sum_{i}^{N} T P_{i} * u\left(T_{i}\right),
$$

wherein

$$
u\left(T_{i}\right)= \begin{cases}1, & T_{i} \text { is executed } \\ 0, & T_{i} \text { is not executed }\end{cases}
$$

Modeling from the perspective of the constraint satisfaction model, when solving, first, select all feasible solutions according to various constraints, and finally obtain the optimal solution of the model according to the optimization objective function.

For the research on the application of constraint satisfaction model to satellite task planning, as early as 1996, Agn et al. [39] proposed a scheduling model based on Valued Constraint Satisfaction Problem (VSCP) in the research on the task planning of SPOT-5 satellite. Flavius Galiber and Stephen A. Dimitrov [40] of France solidified the model framework of satellite mission planning and realized its visualization. Based on constraint satisfaction planning and design, they developed the Generic Resource Event and Activity Scheduler (GREAS) system. In October 2002, NASA proposed in a report [41] that a constraint-based planning system will be developed based on dynamic constraint satisfaction technology for mission planning of aerospace systems (satellites, aircraft, etc.) to improve their automatic planning ability. He [42] of the University of Defense Science and Technology first gave a brief overview of the constraint satisfaction problem, then considered the path constraint and time window constraint, and established a simple constraint satisfaction model with the maximum total task weight as the objective function. In 2018, Guohui [43] established a constraint satisfaction model with risk assessment for the planning of multi-imaging satellites, considered three constraint sets: basic constraints, resource difference constraints, and satellite activity sequence constraints, and took maximizing task support, maximizing resource saving, and balancing resources and task support as optimization strategies, Thus, the hierarchical evaluation system of satellite mission planning scheme with variable weight is established.

In the process of studying satellite mission planning, other scholars have used a constraint satisfaction model or its derived model, but the constraints considered are different from the selected objective function. The constraint satisfaction model is applicable to multitype task planning such as single satellite single load and single satellite multiload. It has a wide range of applications. Only for multiload task planning, it is difficult to use it for modeling because its constraints are difficult to decouple. In addition, the constraints are generally simplified, which is quite different from the actual satellite operation process, so the authenticity of the model is poor.

3.2. Integer Programming Model. An integer programming model is a model that abstracts the problem into an accurate quantitative data relationship and then expresses it in a mathematical form. It can describe the linear constraints in satellite mission planning, and the solution method is relatively simple. It can be solved directly by using existing software tools (such as ILOX CPLEX). Therefore, the integer programming model is also widely used in task planning modeling.

Before introducing the model, we need to describe each parameter in the model, as shown in Table 1.

For the simplified satellite mission planning problem, we give the structure of the integer programming model as follows:

$$
\operatorname{Max} \sum_{i \in T} \sum_{k \in S} p_{i, k} *\left(\sum_{t=1}^{N_{i, k}} x_{i, k, t}\right)
$$


TABLE 1: Temperature and wildlife count in the three areas covered by the study.

(a)

\begin{tabular}{lc}
\hline Set & Meaning \\
\hline$T$ & Task set \\
$S$ & Satellite set \\
$N_{i, k}$ & The number of time windows allowed when \\
task $i$ uses resource $k$
\end{tabular}

(b)

\begin{tabular}{|c|c|}
\hline Parameter & Meaning \\
\hline$p_{i, k}$ & Profit of satellite $k$ performing task $i$ \\
\hline$e_{i, k}$ & Time for satellite $k$ to perform mission $i$ \\
\hline$s_{i, j, k}$ & $\begin{array}{l}\text { Conversion time to continue task } j \text { after } \\
\text { task } i \text { is completed }\end{array}$ \\
\hline$Z$ & $\begin{array}{l}\text { A large integer, usually the length of time } \\
\text { in the planning cycle }\end{array}$ \\
\hline
\end{tabular}

(c)

\begin{tabular}{l}
\hline Variable \\
$x_{i, k, t}=\left\{\begin{array}{lc}1 & \text { Task } i \text { is executed in the } t \text {-th window of resource } k \\
0 & \text { Other }\end{array}\right.$ \\
$y_{i, j}=\left\{\begin{array}{lc}1 & \text { On the same resource, task } i \text { is executed before task } j \\
0 & \text { Other }\end{array}\right.$
\end{tabular}

$$
\begin{gathered}
\text { s.t. } \forall i \in T \sum_{k \in S} \sum_{t=1}^{N_{i, k}} x_{i, k, t} \leq 1, \\
\forall i, j \in T, i \neq j, \forall k \in S y_{i, j}+y_{j, i}+1 \geq \sum_{t=1}^{N_{i, k}} x_{i, k, t}+\sum_{t=1}^{N_{j, k}} x_{j, k, t}, \quad(11) \\
\forall i, j \in T, i \neq j t_{i}-t_{j}+\sum_{k \in S} \sum_{t=1}^{N_{i, k}} x_{i, k, t} *\left(e_{i, k}+s_{i, j, k}\right) \leq Z *\left(1-y_{i, j}\right)
\end{gathered}
$$

Among them, the objective function (9) ensures the maximum total benefit of task execution, the constraint condition (10) ensures that a task can only be executed once at most, the constraint condition (11) logically specifies the sequence of tasks executed on the same resource, and the constraint condition (12) ensures that there can be no intersection during task execution; that is, two tasks cannot be executed on the same resource at the same time.

The research of integer programming model is basically nested in the whole satellite task planning research. In order to solve the problem of single satellite task planning, Song et al. [44] established a nonlinear mathematical programming model based on the visible time window of task planning and aiming at maximizing the benefit of time window and task coverage. In order to solve the problem of multisatellite task planning, $\mathrm{He}$ et al. [45] disassembled it into two problems: multisatellite task allocation and single satellite task planning, and designed a mixed integer programming model for multisatellite task allocation. Yunfeng and Xiaoyue [46] established single data transmission task model and satellite data transmission task model for multisatellite data transmission task scheduling problem. Wei [47] analyzed the advantages and disadvantages of traditional mathematical programming and constraint satisfaction optimization programming and found that they can complement each other to a certain extent. The characteristics of integer programming model are used to deal with the "optimization" characteristics (i.e., objective function), and the constraint satisfaction model is used to quickly solve a feasible solution, and finally, a hybrid model is established.

However, the integer programming problem itself is difficult to solve, especially when the scale of the problem increases; if there is no appropriate branch and bound strategy, the solution efficiency will be very low. In addition, the model can not deal with some nonlinear constraints.

3.3. General Problem Model. In addition to the constraint satisfaction model and integer programming model, there are other general problem models, such as backpack model, directed graph model, and multiagent model.

3.3.1. Backpack Model. The backpack model is a classical dynamic programming model used to solve operational research problems. It can be simply summarized as a backpack with $n$ items and a capacity of $M$. The cost of item $i$ is $c_{i}$ and the value is $w_{i}$. We need to know which items can be loaded into the backpack to maximize the total value. Compared with the satellite task planning problem, it can be described as $n$ tasks to be observed and the observation sequence with a total amount of $m$ on the satellite. The power consumption of the observation task $i$ is $p$, the storage consumption is $d_{i}$, and the observation income is $w_{i}$. Therefore, the maximum income of adding the tasks to be observed to the observation sequence under the constraints of total power and total storage is solved. The model comparison of the two problems is shown in Figure 8.

In the research on the backpack problem model, Pemberton and Galiber [40] analyzed the task planning problem of SPOT-5 satellite and transformed the imaging satellite problem into a backpack problem model in 2001, so that the backpack problem model can be used to solve the task planning problem of imaging satellite. In 2008, Wang et al. [15] regarded the imaging task to be observed as an "item" and the reconnaissance time window as a "backpack" with multiple attributes. They proposed the concept of "Compatibility List" to describe the complex correspondence between items and backpacks and established a multidimensional dynamic backpack model, which reduced the satellite task planning problem to a multiobjective optimization problem. In 2012, Jung-Hyun et al. [48] regarded the task scheduling problem as a backpack problem, adopted dynamic programming, and greedy algorithm 


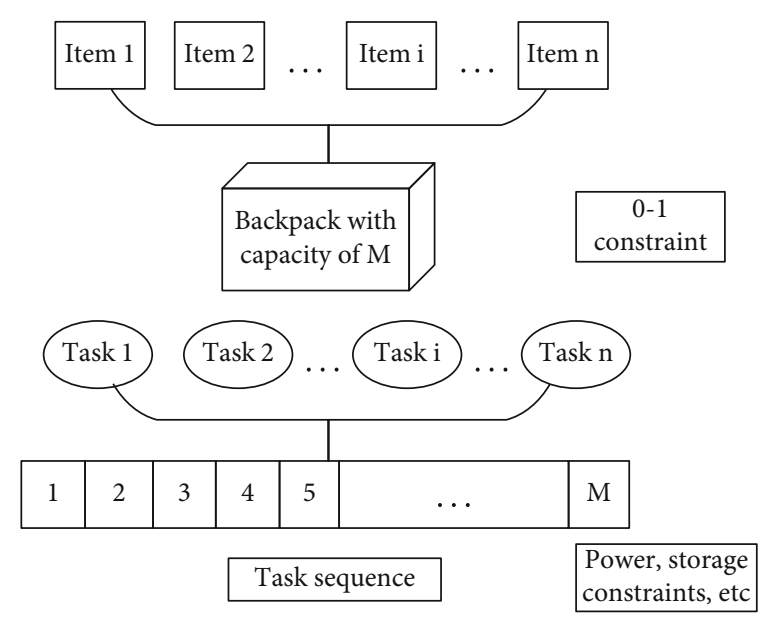

FIGURE 8: Comparison between satellite mission planning problem and backpack problem model.

for multisatellite cooperative task planning and scheduling, and compared and analyzed the two algorithms.

3.3.2. Directed Graph Model. The directed graph model, also known as Bayesian network, is a probability graph model. The nodes in the directed graph model represent random variables, which can be observable variables, hidden variables, unknown parameters, and so on. If there is a causal relationship between the two variables, use an arrow to connect them. If two nodes are connected by a single arrow, it means that one node is "parents" and the other is "children," and the two nodes will produce a conditional probability value.

When the directed graph model is applied to the modeling of satellite task planning, the earth imaging task can be regarded as the vertex of a directed graph, and the attributes of the imaging task, such as task start time, task end time, observation load required by the task, and imaging required resolution, are added to the attribute tuple of the vertex, and the vertices are sorted according to the observation time of the task. In addition to the data transmission tasks between satellites and the virtual start and end points, a time ordered directed graph is constructed, as shown in Figure 9.

The directed graph model can intuitively represent the task scheduling problem of a single imaging satellite, but when multiple imaging satellites are considered, the directed graph model will increase the difficulty of problem analysis. Zhang et al. [49] established the corresponding time-series acyclic digraph model by using graph theory to measure the imaging work with multicriteria and proposed the time-series multicriteria shortest path algorithm on this basis. Wang et al. [50] adopted the preallocation method to transform multisatellite task planning into single satellite task planning and used the directed graph model to describe the integrated task scheduling problem of imaging satellites. Then, based on the directed graph model, the task scheduling problem of a single imaging satellite is transformed into the imaging path search problem in the directed graph. On this basis, a multitarget imaging path search algorithm based

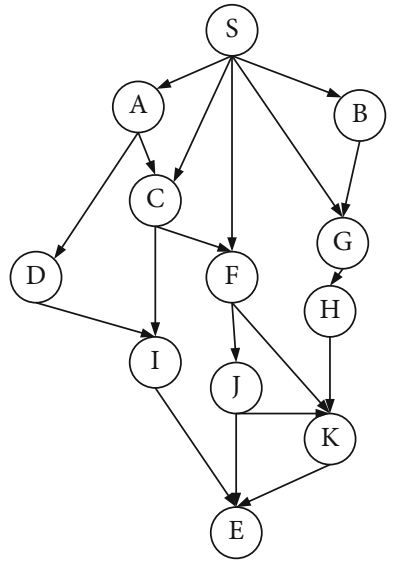

FIGURE 9: Schematic diagram of directed graph model.

on the NSGA2 algorithm is proposed to solve the task scheduling problem of each imaging satellite.

3.3.3. Agent Model. Agent modeling is an effective method for complex system modeling in distributed computing environment. Modeling and simulation based on multiagent system (MAS) realizes the overall modeling of the system by modeling the interaction between entities and obtains the overall "emergence" behavior of the system. It is a bottomup modeling method, which abstracts the entities in the system and environment into reactive and active agents, and then assembles them with appropriate MAS architecture, so as to establish the simulation model of the whole system and realize the simulation of the overall behavior of the system. At present, the modeling and simulation technology based on MAS has become one of the more effective methods for the research of complex system problems. The task planning of satellite, especially multisatellite distributed system, involves many elements and multiple systems. It is suitable to use multiagent system for structural design and modeling. The agent model of the whole process of satellite mission planning is shown in Figure 10.

For multisatellite task planning, Schetter et al. [51] construct a multisatellite task negotiation and allocation method with a hierarchical structure from the perspective of autonomous system in 2004, based on multiagent theory, and expressed it as four different levels of agents according to the intelligence level of each satellite. According to the distributed and intelligent characteristics of distributed imaging satellite system, Zhang et al. [52] studied the distributed imaging satellite system by using the idea of agent and multiagent system in 2006 and proposed a highly reliable MAS structure of distributed imaging satellite system and a hierarchical hybrid structure model of intelligent imaging satellite agent. The honest contract network protocol for distributed satellite system Constellation task planning is used, and the rule-based bidding process is realized to solve it. In 2012, Hao et al. [53] established an agent model for multipayload earth observation satellite mission driven planning and proposed a multilevel agent hybrid collaborative planning algorithm based on contract network protocol and blackboard model. Until 2019, Weiyi and Xiaolu [54] studied 


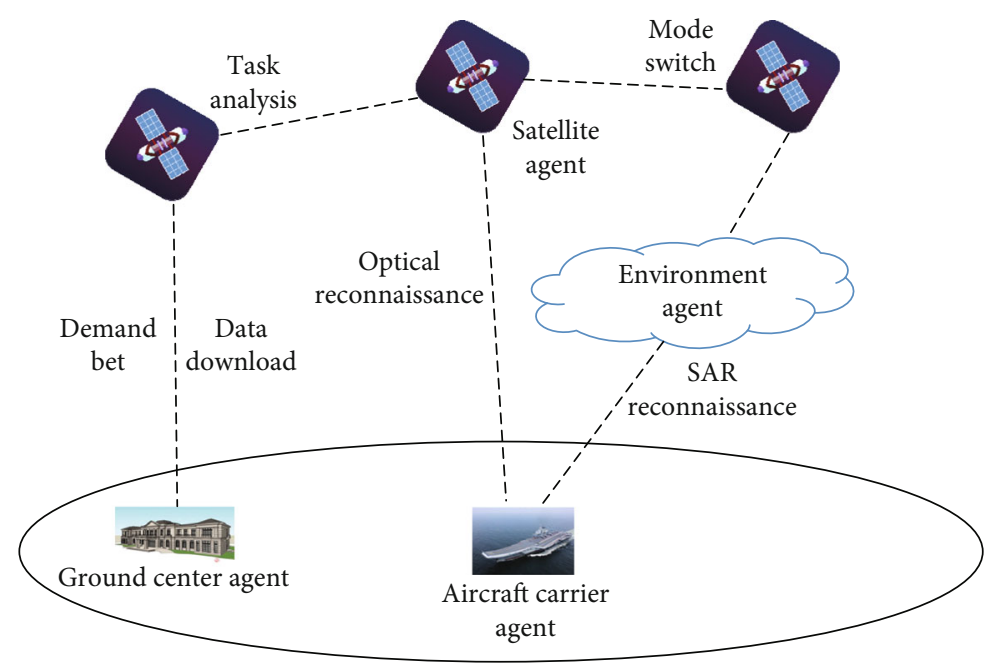

FIgURE 10: Agent model of the whole process of task planning.

the problem of multisatellite autonomous collaboration and task planning, established a multiagent model of the problem, and introduced a dynamic centralized distributed architecture. On the basis of the architecture, they introduced the contract network protocol as the interaction and negotiation mechanism of collaborative task planning and improved and expanded it in two aspects: contract network process and bid evaluation strategy. A dynamic contract network algorithm for multiautonomous satellite cooperation is also designed. Other scholars [14, 55-57] have also studied the application of agent model to satellite mission planning.

Different from other models, the multiagent model has a certain degree of intelligence and autonomy. It is suitable for on-board dynamic task planning in an autonomous satellite system. It can deal with emergencies that traditional static models are difficult to adapt to dynamic arrival. However, most of the current agent models are centralized masterslave architecture. The master satellite agent has a high degree of intelligence, and other satellites depend on it. The reliability of the system is poor, and it does not reflect the adaptability of the distributed satellite system to the change of dynamic observation environment. Therefore, it is necessary to study a new distributed intelligent agent model, so that the satellite system can complete the dynamic task planning to adapt to the dynamic environment.

3.4. Summary of This Chapter. This section mainly combs three types of models commonly used in imaging satellite mission planning research, namely, constraint satisfaction model, integer programming model, and general problem model. The general problem model includes the backpack model, directed graph model, and multiagent model. Different models have different emphases. Constraint satisfaction models have strong description ability. They mainly describe the constraints in planning problems clearly, making the representation of variables and constraint relations concise and clear. The integer programming model is easy to solve, and the existing software tools can be used, but it can only describe the linear constraints in the programming problem. The general problem model generally transforms the satellite mission planning problem into other problems, which makes the model universal. However, in the process of modeling, some constraints need to be reasonably simplified, which is difficult to achieve a comprehensive description of the problem.

\section{Algorithm for Solving}

The establishment of the model is to describe the problem in mathematical language or computer language, and then, the solution of the problem also needs to solve the model. Based on the satellite mission planning model, the planning algorithm can solve the model and optimize the results. Therefore, the design of algorithm is also an important link in solving satellite mission planning problem. When solving the satellite task planning model, because multisatellite task planning is a complex problem with multiple tasks to be observed, multiple constraints, and multiple optimization functions, the solution space is also a huge set; it is difficult to traverse. Therefore, the most used algorithms are intelligent search algorithms and heuristic algorithms in approximate algorithms. A few small-scale satellite mission planning problems will also use some accurate algorithms. In recent years, artificial intelligence has developed rapidly. Artificial intelligence algorithms such as machine learning and deep learning have also been applied to solve task planning models. The algorithms applied to satellite mission planning can be roughly classified as follows, as shown in Figure 11.

This chapter will start with heuristic algorithm, metaheuristic algorithm, exact algorithm, and artificial intelligence algorithm; study the theoretical basis, algorithm structure, and application advantages and disadvantages of different algorithms; and give the applicable solution model of the algorithm, so as to provide reference for the solution of satellite task planning problem.

4.1. Heuristic Algorithm. The heuristic algorithm is a search algorithm proposed relative to the optimization algorithm. It can give the feasible solution of the problem in a certain time 


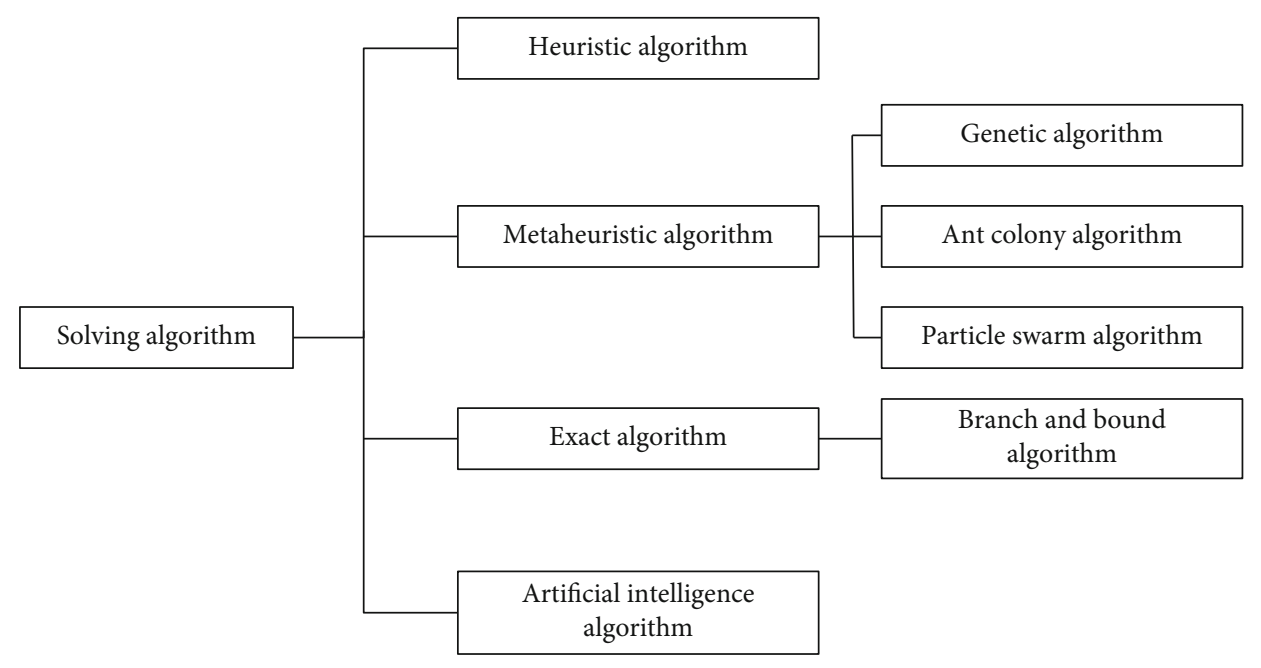

FIGURE 11: Algorithm for solving satellite mission planning.

and space, but it is not necessarily the optimal solution of the problem. Heuristic algorithms are special and generally rely on a specific problem, and there is no general algorithm structure.

As early as 2007, Wang et al. [58] considered the complex environmental constraints in a satellite work, such as the inability to observe the target point caused by cloud cover, the addition of temporary observation tasks, and changes in satellite observation attributes. They use a unified form, that is, inserting new tasks, to describe these problems, propose a rule-based heuristic algorithm, and design a maximum contraction heuristic rule and a minimum occupancy heuristic rule for insertion.

In 2014, Wang et al. [36] proposed two heuristic algorithms based on time window congestion and task overlap for dynamic task planning, considering timing constraints and task constraints and explained the different characteristics and advantages of the two heuristic algorithms in comparative experiments.

Until 2020, Han et al. [59] transformed the task replanning problem of imaging satellite into the temporary insertion problem of emergency task and designed a three-level heuristic task sequence solution method of direct task insertion, local task adjustment, and task deletion.

It can be seen that the heuristic search algorithm is generally used for satellite dynamic task planning. Because dynamic task planning requires high planning time and needs to replan the existing task sequence in a short time, it is difficult to use the optimization algorithm to obtain the optimal solution, but it also exposes the disadvantages of inaccurate results and nonoptimization.

\subsection{Metaheuristic Algorithm}

4.2.1. Evolutionary Algorithms. The evolutionary algorithm is a bionic algorithm cluster inspired by the biological evolution of nature and simulating the law of biological genetic evolution. The genetic algorithm and its improved algorithm are most used. The genetic algorithm is simple, universal, and robust. The main steps of the algorithm are shown in Figure 12.

When solving the constraint satisfaction model of task planning, Wei et al. [60] proposed an immune ant colony genetic hybrid algorithm (IA-COGA). They use the solution generated by the ant colony algorithm as the initial population of the immune genetic algorithm and take the global optimal solution in the ant colony algorithm as the vaccine. Compared with the general genetic algorithm, the IA-COGA algorithm has higher accuracy and faster convergence speed.

When solving the integer programming model of task planning, Farui [61] used the improved genetic algorithm. According to the individual's satisfaction of time partial order constraints and resource constraints, he first divided the individual into three types of states, designed appropriate crossover and mutation operators, respectively, and then used task partial order sorting and individual classification to exclude some nonoptimal individuals in advance and reduce the search space. Thus, the computational efficiency of the algorithm is improved.

When solving the mathematical model of multisatellite task planning, Song et al. [62] designed and proposed an improved genetic algorithm containing two optimization strategies, including global optimization and local optimization, which can dynamically adjust the number of individuals in the population and improve the solution speed.

4.2.2. Swarm Intelligence Algorithm. In 1989, Kennedy [63] first proposed the concept of swarm intelligence. The swarm intelligence optimization algorithm is a random search evolutionary algorithm based on probability. It mainly simulates the group behavior of insects, herds, birds, and fish. The main behavior of these groups is foraging. They will find food in a cooperative way and then constantly exchange food information, so as to find more food more quickly. The swarm intelligence algorithm is a computing technology based on the behavior law of biological groups, which is used to solve distributed problems. It is mainly used in combinatorial optimization, image processing, data mining, and 


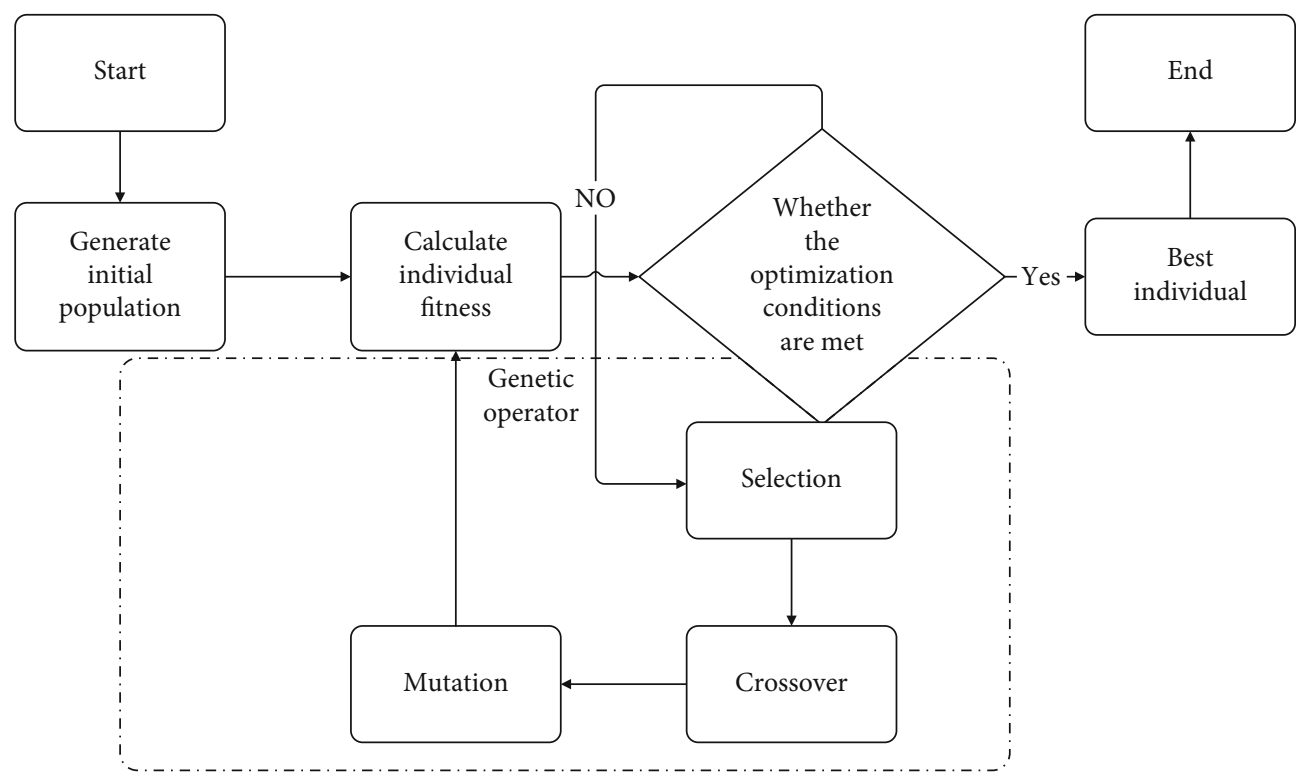

FIgURE 12: Flow chart of main steps of genetic algorithm.

other fields [64]. Among them, the most classical swarm intelligence algorithms are ant colony algorithm and particle swarm optimization algorithm.

(1) Ant Colony Algorithm (ACO). The ant colony algorithm is a kind of swarm intelligence algorithm. In 1991, Colorni et al. [65] proposed such an evolutionary algorithm to simulate the path finding of ant population. It mainly includes two parts: path construction and pheromone update. Combined with the task planning problem, the basic idea of ant colony algorithm is that firstly, each artificial ant represents a task planning sequence and carries out single track optimal synthesis on the basis of each task planning scheme to obtain the optimal synthetic observation scheme and income under the task allocation scheme and takes the synthetic observation income as the evaluation of the "path," strengthen the pheromone on the better "path," so as to guide the ants to converge to the better "path," and finally get the optimized task allocation scheme. The flow of the algorithm is shown in Figure 13.

Bai et al. [66] proposed an adaptive ant colony algorithm to solve the task allocation problem by using the positive feedback and strong search performance of ant colony algorithm. Through the task synthesis results under multiple task allocation schemes, the advantages and disadvantages of the task allocation scheme are fed back, and the ant colony is guided to search for a better task allocation scheme, so as to obtain the optimized allocation scheme and synthesis scheme. Through adaptive parameter adjustment and pheromone smoothing strategy, the balance between global search and fast convergence is realized.

Based on the constraint satisfaction model of planning and scheduling, Derong [67] improved and solved the basic ant colony algorithm from the aspects of pheromone distribution diversity, pheromone update strategy, state transition, and other mechanisms.

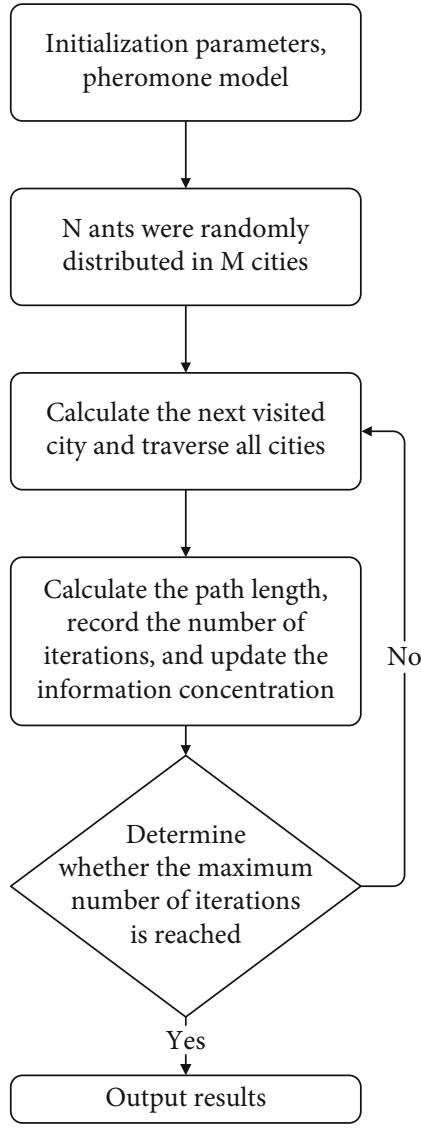

Figure 13: Basic flow diagram of ant colony algorithm.

According to the problem characteristics of smart satellite task planning, Yuning et al. [68] introduced the knowledge learning and pheromone restriction strategy into the traditional ant colony algorithm and proposed the task path processing process, so as to generate a scheduling scheme to 
evaluate the advantages and disadvantages of the path and feed it back to the ant path search stage, so as to improve the solution efficiency.

For the ant colony algorithm, the cooperative individuals are distributed, there is no central control, and it is robust. Cooperation can be carried out through communication between individuals, which is scalable [69]. However, the ant colony algorithm also has the disadvantages of poor flexibility and applicability, and it is easy to stagnate in the calculation process.

(2) Particle Swarm Optimization (PSO). The particle swarm optimization algorithm is a random search algorithm based on swarm intelligence developed by simulating the foraging behavior of animals and birds. Kennedy et al. [70] first proposed this concept in his article in 1995. For particle swarm optimization, the solution of each optimization problem is a bird in the search space, also known as "particle." As a biological evolutionary algorithm, the particle swarm optimization algorithm and genetic algorithm have many similarities. For example, both need population initialization, use fitness to evaluate the system, and carry out certain random search. However, the particle swarm optimization algorithm has no genetic operation and only needs to follow the current optimal particle to search in the solution space. The flow of particle swarm optimization algorithm is shown in Figure 14.

When solving the constraint satisfaction model of satellite task planning, Wei and Xueqing [71] used a discrete particle swarm optimization algorithm to effectively solve the problem of multisatellite task planning. Chen [72] and others proposed a multisatellite real-time playback data transmission scheduling algorithm based on quantum discrete particle swarm optimization (QDPSO) for the data transmission scheduling problem of earth observation satellites and designed the longest compatible transmission chain mutation operator to improve the performance of the algorithm.

The particle swarm optimization algorithm has strong universality and does not depend on problem information. It has memory ability in group search, can retain the optimal information of local individual and global population, and can use individual local information and group global information to guide the next search of the algorithm. However, there is no mathematical proof of the convergence and convergence rate estimation of particle swarm optimization algorithm, and the research on its theoretical and mathematical basis is not enough. The particle swarm optimization algorithm can not guarantee the optimal solution in theory. Its application range in solving optimization problems is limited, especially for discrete combinatorial optimization problems; its theoretical modeling is still in its infancy.

4.2.3. Local Search Algorithm. The local search algorithm is a metaheuristic algorithm corresponding to the global search algorithm. Starting from the initial solution, it constantly searches the domain space and selectively moves like the optimal solution. In the application of satellite mission plan-

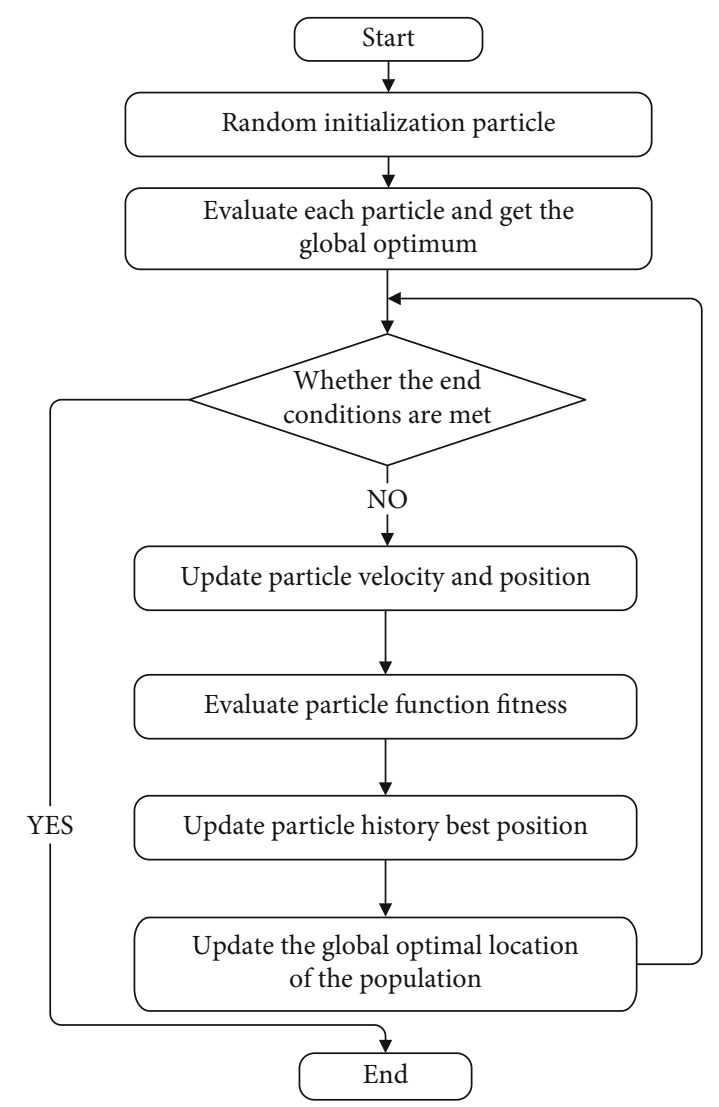

FIGURE 14: Flow diagram of particle swarm optimization algorithm.

ning, the most common local search algorithms are tabu search algorithm and simulated annealing algorithm.

(1) Tabu Search Algorithm. The tabu search algorithm was first proposed by Professor Glover [73, 74] of the United States in 1986, and the principle of the algorithm was introduced. The difference between the tabu search algorithm and other algorithms is that in order to improve the deficiency that local neighborhood search is easy to fall into local optimum; it constructs a short-term circular memory table, that is, tabu table, which stores the searched local optimum in the tabu table. In the next search, it will no longer search or selectively search the information in the tabu table, so as to jump out of the local optimum, so as to finally achieve global optimization.

For the model solution of satellite mission planning, Chen et al. [75] improved the basic tabu search algorithm, alternately used the improved neighborhood and adjusted neighborhood for the problem, and proposed a variable neighborhood tabu search algorithm for model solution based on the improved neighborhood. Based on the constraint satisfaction model of task planning, $\mathrm{He}$ [42] proposed the concept of backward free time and used a specific example to solve the model with tabu search algorithm. When solving the satellite TT and C task scheduling problem, Blöchliger and Zufferey [76] select the tabu search algorithm with variable tabu length and selects and designs the domain structure. 
(2) Simulated Annealing Algorithm. The simulated annealing algorithm is a probability-based algorithm that simulates the physical annealing process of solids. It was first proposed by N. Metropolis [77] in 1953. The simulated annealing algorithm starts from a high initial temperature, with the continuous decline of temperature parameters, combined with the characteristics of probability jump, and randomly looks for the global optimal solution of the objective function in the solution space. The simulated annealing algorithm is a random algorithm, which can not necessarily find the global optimal solution. However, it can quickly find the approximate optimal solution of the problem and has a certain application in satellite mission planning.

Yang [20] designed the simulated annealing algorithm flow when solving the multistar multiobjective task planning problem and solved it according to the stability criterion and Metropolis criterion. According to Huan et al. [78], based on the mixed integer programming model of static task planning of electronic reconnaissance satellite, the classical simulated annealing algorithm is used to effectively solve the task planning problem of electronic reconnaissance satellite for fixed targets. Chao and Yanbin [79] designed a hybrid genetic simulated annealing algorithm based on similarity and aggregation based on the principle of the original simulated annealing algorithm on the basis of constructing the combined optimization model of agile satellite task planning. Using the global search ability of the genetic algorithm is conducive to changing the disadvantage that the simulated annealing algorithm is easy to fall into local minimum points and finding better results.

4.3. Exact Algorithm. As the name suggests, the exact algorithm refers to the algorithm that can accurately find the optimal solution. It is generally used for small-scale planning problems. The commonly used accurate algorithms include branch and bound algorithm, dynamic programming algorithm, and cut plane method. Because satellite mission planning is mostly a large-scale observation mission planning problem, this kind of algorithm is less used in terms of computational efficiency, but it still has certain guiding significance to deal with some single satellite and small-scale problems.

The branch and bound algorithm was first proposed by land [80] in 1960. It is a search and iterative method. It selects different branch variables and subproblems to branch. It is most commonly used to solve integer programming problems. Therefore, it is often used in integer programming models for satellite mission planning.

On the basis of considering the uncertain constraints of cloud cover, Valicka et al. [81] established a mixed integer programming model and solved it by branch and bound algorithm. Xiaogeng [82] designed a branch and bound algorithm for agile satellite scheduling problem under timing constraints, using the depth first search principle, assuming that the geographical sequence of tasks in the offline direction is the order of variable expansion, and reasonably simplified some relevant constraints.

4.4. Artificial Intelligence Algorithm. In addition to the traditional optimization algorithms and search algorithms, the vigorous development of artificial intelligence also promotes the intelligent development of task planning. In particular, satellite online mission planning needs a certain degree of intelligence.

At present, the use of artificial intelligence method to solve the task planning problem is in its infancy. As early as 2015, Song et al. [83] extracted the characteristics of imaging tasks based on the classical scheduling model of imaging satellites and used the back propagation (BP) neural network integration algorithm with variable hidden layer nodes to solve the scheduling problem of imaging tasks. In 2018, Li and Chen [84] combined the supervised learning method with the heuristic search algorithm and used the neural network to calculate the scheduling priority of each task, and then the heuristic search algorithm successively inserted the observation tasks into the observation scheme according to the scheduling priority of the task, which enhanced the optimization ability of the heuristic search algorithm. In the same year, Haijiao [85] established the satellite realtime service model based on Markov decision process and the satellite online scheduling model based on Markov game for the satellite online scheduling problem in centralized structure and distributed structure, respectively, and solved it by using the satellite online scheduling algorithm based on reinforcement learning. Y. Min and Z. Min [86] constructed a task flow autonomous scheduling decisionmaking system by using a fuzzy neural network in the process of studying small satellite task autonomous planning. In 2019, Wang et al. [87] mapped the satellite task planning problem into a dynamic random knapsack problem and used $\mathrm{A} 3 \mathrm{C}$ (asynchronous advantage actor critical) reinforcement learning algorithm to decide whether each observation task should be inserted into the observation scheme in real time. Experiments show that this method is superior to the first come first serve greedy algorithm in optimization.

The application of artificial intelligence in task planning and resource scheduling effectively improves the solution efficiency and optimization performance of task planning algorithm. However, in practice, the planning problem of multisatellite, multiload, and multitask has complex constraints. How to transform the planning problem into satellite autonomous intelligent online real-time planning still needs further application of artificial intelligence methods.

4.5. Summary of This Chapter. By comparing the principle and algorithm structure of heuristic algorithm, metaheuristic algorithm, exact algorithm, and artificial intelligence algorithm, it is found that the local search algorithm can get a better solution with the shortest time, but the types of constraints are limited; considering all constraints, the genetic algorithm has the best performance, but it is difficult to code the implementation of the whole solution algorithm; the exact solution is the easiest to use, and the optimal solution of the programming problem can be obtained, but it is not satisfactory to deal with the combinatorial explosion problem. An efficient algorithm matching the problem model must be specially designed; the artificial intelligence algorithm improves the intelligence of satellite, but it still 
needs to learn with traditional algorithms in order to have excellent solution efficiency and optimization performance.

\section{Future Research Focus}

Through the understanding and research of satellite mission planning in recent years, I think the following points will be the focus of future research in this field.

\subsection{Mission Planning of Large-Scale Distributed Satellite} System. A distributed satellite system is a mission architecture composed of multiple space spacecraft. Compared with a single satellite, it can increase flexibility and robustness and improve continuous real-time global coverage. The aerospace industry is also experiencing a continuous shift from large and expensive satellites to small, cheap, and massproduced satellites [88]. For example, SpaceX proposed by the United States plans to build a star chain network composed of about 12000 satellites in space from 2019 to 2024, of which 1584 will be deployed in low earth orbit 550 kilometers above the earth. Such a large-scale satellite system also poses a new great challenge to the mission planning of the whole system. The huge constraints make the traditional programming model difficult to describe accurately. The exponential growth of data also makes the scale of the algorithm larger, the execution time longer, and the solution efficiency lower. Therefore, how to plan the mission of largescale distributed satellite system will be a challenge in the future.

5.2. Real-Time Intelligent Mission Planning on Satellite. Most of the existing satellites are deterministic ground planning. During the operation of satellites, they can only mechanically execute the task instructions uploaded from the ground. However, many uncertain problems will be encountered in the actual operation of the satellite. For example, during the operation of the optical reconnaissance satellite, the change of meteorological conditions has a great impact on its work. If it continues to perform a fixed task after being blocked by clouds, it will lead to task failure and waste observation resources. Taking the satellite fault problem as an example, if the components fail during the operation of the satellite, the interruption of a load will not affect the satellite operation, but affect the satellite earth observation, and the mission will still fail if it continues to be executed according to the original mission instructions.

On the other hand, in some emergencies, there are high practical requirements for satellite observation and data transmission. For example, in the process of earthquake relief, the rational use of satellites can make the rescue more efficient. However, the situation in the disaster area is complex and changeable, and a fixed task instruction can not grasp the real-time dynamics of the dangerous situation in the disaster area.

This requires future research to focus on the following: (1) satellite real-time intelligent mission planning. On the basis of fault diagnosis and environmental sensing, satellites can analyze uncertain factors, establish models, and modify mission plans to reduce losses. (2) In the face of unexpected emergency tasks, the satellite can independently decide whether to execute, establish a task planning model and select reasonable task execution time and execution resources, so as to obtain the maximum benefit and minimum resource consumption while completing the task.

5.3. System-Wide Comprehensive Planning. Satellite task execution is a multifactor and multiprocess system, not just a simple task scheduling. We need to make reasonable planning for many satellite activities such as task reception, task selection, task distribution, intersatellite information interaction, and data transmission. Therefore, future task planning is system planning. This requires future research to focus on the following: (1) multiple activities should be considered in the establishment of the model, and the constraints between activities should be supplemented and improved; (2) more general and simple algorithms should be studied to reduce the solution complexity and improve the solution efficiency; (3) establish the system planning evaluation model and further optimize the model.

In addition, there must be many research directions, such as intelligent semantic understanding of the task requirements submitted by users, further improvement of on-board computing capacity, and self-feedback of task completion to the satellite, so that the satellite can optimize observation and improve task execution ability at the next transit.

\section{Summary}

Satellite mission planning is the core module of satellite system, and the planning level is an important factor affecting the efficiency of satellite work. The research on imaging satellite mission planning has lasted for decades. With the further development of aerospace industry, the research on mission planning will enter the next development peak. This paper systematically combs the major problems in the research of imaging satellite mission planning in recent years and summarizes the advantages and disadvantages of related technologies.

The main work is as follows: (1) firstly, according to different classification standards, the satellite task planning problem is divided into single satellite single load task planning, multisatellite single load task planning, multisatellite multiload task planning, point target task planning, regional target task planning, static planning, and dynamic planning. The characteristics, key technologies, and development direction of each planning problem are analyzed; (2) this paper focuses on the analysis of various models established in the research of task planning and gives a detailed description of the modeling process, model characteristics and application scope of the model; (3) this paper summarizes the algorithms used in the process of model solving and expounds the principle, algorithm architecture, advantages and disadvantages, applicable models, and so on; (4) finally, based on the comprehensive consideration of the research in recent years, this paper puts forward some thoughts on the development of task planning in the future. 


\section{Conflicts of Interest}

The authors declare that there is no conflict of interest regarding the publication of this paper.

\section{References}

[1] J. Jun, "A review of remote sensing imaging observation technology," Northeast Surveying and Mapping, vol. 4, pp. 7-8, 2000.

[2] L. Qiyuan and Y. Ying, "A method of literature catalog information mining technology and the implementation of its software SATI: a case study of Chinese and foreign library intelligence," Journal of Information Resource Management, vol. 2, no. 1, pp. 50-58, 2012.

[3] Y. Li, R. Wang, and M. Xu, "Rescheduling of observing spacecraft using fuzzy neural network and ant colony algorithm," Chinese Journal of Aeronautics, vol. 27, no. 3, pp. 678-687, 2014.

[4] N. G. Hall and M. J. Magazine, "Maximizing the value of a space mission," European Journal of Operational Research, vol. 78, no. 2, pp. 224-241, 1994.

[5] E. Bensana, G. Verfaillie, J. C. Agnese, N. Bataille, and D. Blumstein, "Exact \& inexact methods for daily management of earth observation satellite," Space Mission Operations and Ground Data Systems-Spaceops '96, vol. 394, 1996.

[6] M. Vasquez, J. Hao, and P. Besse, "A "logic-constrained" knapsack formulation and a tabu algorithm for the daily photograph scheduling of an earth observation satellite," Computational optimization and applications, vol. 20, no. 2, pp. 137-157, 2001.

[7] W. J. Wolfe and S. E. Sorensen, "Three scheduling algorithms applied to the earth observing systems domain," Management Science, vol. 46, no. 1, pp. 148-166, 2000.

[8] S. Lee, W. C. Jung, and J. Kim, "Task scheduling algorithm for the communication, ocean, and meteorological satellite," ETRI Journal, vol. 30, no. 1, pp. 1-12, 2008.

[9] S. Peng, H. Chen, J. Li, and N. Jing, "A heuristic method for single satellite observation and transmission tasks planning," in 2017 13th international conference on natural computation, fuzzy systems and knowledge discovery (icnc-fskd), pp. 29252932, Guilin, China, 2017.

[10] X. Chu, Y. Chen, and Y. Tan, "A branch and bound algorithm for agile earth observation satellite scheduling," Advances in Space Research, vol. 2017, no. 9, pp. 1-15, 2017.

[11] G. Yuan, On-Line Dynamic Planning of Missions for Ground Imaging of Agile Remote Sensing Satellites, Harbin Engineering University, 2019.

[12] B. Du, S. Li, Y. She, W. Li, H. Liao, and H. Wang, "Area targets observation mission planning of agile satellite considering the drift angle constraint," Journal of Astronomical Telescopes, Instruments, and Systems, vol. 4, no. 4, 2018.

[13] Y. Miao, I. Hwang, M. Liu, and F. Wang, "Adaptive fast nonsingular terminal sliding mode control for attitude tracking of flexible spacecraft with rotating appendage," Aerospace Science and Technology, vol. 93, article 105312, 2019.

[14] C. Wang, J. Li, N. Jing, J. Wang, and H. Chen, "A distributed cooperative dynamic task planning algorithm for multiple satellites based on multi-agent hybrid learning," Chinese Journal of Aeronautics, vol. 24, no. 4, pp. 493-505, 2011.
[15] L. Wang, Y. Wang, K. Chen, and H. Zhang, "Optimization of regional coverage reconnaissance satellite constellations by NSGA-II algorithm," in 2008 International Conference on Information and Automation, Changsha, 2009.

[16] H. Hao, W. Jiang, and Y. Li, "Improved algorithms to plan missions for agile earth observation satellites," Journal of Systems Engineering and Electronics, vol. 25, no. 5, pp. 811821, 2014.

[17] S. Nan, Research and Application of Multi-Satellite Distributed Collaborative Mission Planning Technology, University of Chinese Academy of Sciences, 2015.

[18] Z. Yingen, Research on Autonomous Mission Planning Model and Algorithm of Multi-Imaging Satellites, Harbin Institute of Technology, 2017.

[19] G. Yu-hua, J. Ning, L. Jun, and W. Jun, “A comparison of iterative repair strategies for earth observing satellites imaging scheduling," in 2008 Ninth ACIS International Conference on Software Engineering, Artificial Intelligence, Networking, and Parallel/Distributed Computing, Phuket, Thailand, 2008.

[20] F. Yang, Research on Multi-Satellite and Multi-Station Earth Observation Mission Planning Technology, Xidian University, 2010.

[21] Q. Pan, Z. Zhou, R. He, and L. Xing, "Modeling research on joint scheduling of multiple imaging satellites with multiple sensor," Science Technology and Engineering, vol. 11, no. 29, pp. 7174-7178, 2011.

[22] L. Hao, Research on Coordinated Mission Planning Method of Multi-Load Electromagnetic Detection Satellites, National University of Defense Technology, 2012.

[23] L. Yin, Research on Target Access Calculation and Task Scheduling Method of Multi-Load Earth Observation Satellite, National University of Defense Technology, 2012.

[24] L. Longmei, Research on Key Technologies of Multi-Aircraft and Multi-Load Earth Observation Mission Planning, National University of Defense Technology, 2013.

[25] Y. Z. Geng, Y. N. Guo, C. J. Li, and L. I. WB, “Optimal mission planning with task clustering for intensive point targets observation of staring mode agile satellite," Control and Decision, vol. 35, no. 3, pp. 613-621, 2020.

[26] M. Lemaître, G. Verfaillie, F. Jouhaud, J. M. Lachiver, and N. Bataille, "Selecting and scheduling observations of agile satellites," Aerospace Science and Technology, vol. 6, no. 5, pp. 367-381, 2002.

[27] H. Renjie, B. Baocun, C. Yingwu, and T. Yuejin, "Multi-satellite mission planning for environmental and disaster monitoring satellite system," in SpaceOps 2008 conference, Heidelberg, Germany, 2008.

[28] Y. Jing, X. Jinjun, and Y. Longjiang, "Study of one-orbit multistripes splicing imaging for agile satellite," Spacecraft Engineering, vol. 24, no. 2, pp. 27-34, 2015.

[29] L. Jianyin, The Research on Multi-Satellite Scheduling Method Oriented to Forest Resource Observation, Central South University of Forestry and Technology, 2018.

[30] Y. Jingyu, Research on Multi-Satellite Scheduling Method Oriented Regional Target Observation, Hefei University of Technology, 2020.

[31] T. Schiex, Solution Reuse in Dynamic Constraint Satisfaction Problems, Springer, Berlin Heidelberg, 1999.

[32] J. Cpa and L. Ggb, On the Need for Dynamic Scheduling of Imaging Satellites, 2012. 
[33] Y. Liu, Y. Chen, and Y. Tan, "A model and method of multisatellite dynamic scheduling with new tasks arriving," Systems Engineering Theory and Practice, vol. 4, pp. 35-41, 2005.

[34] J. Wang, J. Li, and Y. Tan, Snpd 2007: Eighth Acis International Conference on Software Engineering, Artificial Intelligence, Networking, and Parallel/distributed Computing, Vol 1, Proceedings. 10662 Los Vaqueros Circle, Po Box 3014, Los Alamitos, Ca 90720-1264, Ieee Computer Soc, USA, 2007.

[35] R. Grasset-bourdel, G. Verfaillie, and A. Flipo, "Planning and replanning for a constellation of agile earth observation satellites," in Proc. of the ICAPS-11 Workshop on "Scheduling and Planning Applications»(SPARK-11), Freiburg, Germany, 2011.

[36] M. Wang, G. Dai, and M. Vasile, "Heuristic scheduling algorithm oriented dynamic tasks for imaging satellites," Mathematical Problems in Engineering, vol. 2014, no. 5, p. 11, 2014.

[37] W. Chaochao, Research on Imaging Satellite Emergency Dispatch Problem, Hefei University of Technology, 2019.

[38] D. A. Huffman, "Impossible objects as nonsense sentences," Machine Intelligence, vol. 6, pp. 295-324, 1971.

[39] J. C. Agnese, N. Bataille, D. Blumstein, and E. Bensana, Exact and Approximate Methods for the Daily Management of an Earth Observation Satellite, 1996.

[40] J. C. Pemberton and F. Galiber, "A constraint-based approach to satellite scheduling," DIMACS Series in Discrete Mathematics and Theoretical Computer Science, vol. 57, pp. 101-114, 2001.

[41] J. Rintanen and H. Jungholt, "Numeric state variables in constraint-based planning," in European Conference on Planning, Berlin, Heidelberg, 1999.

[42] R. He, "Design and implementation of satellite global survey mission scheduling system," Computer Engineering \& Applications, vol. 42, no. 21, p. 9, 2006.

[43] L. Guohui, Robust Mission Planning Model and Algorithm for Multi-Imaging Autonomous Satellites, Harbin Institute of Technology, 2018.

[44] D. Song, A. Stappen, and K. Goldberg, "An exact algorithm optimizing coverage-resolution for automated satellite frame selection," in IEEE International Conference on Robotics and Automation (icra), New Orleans, LA, USA, 2004.

[45] R. He, P. Gao, B. C. Bai, J. F. Li, F. Yao, and L. N. Xing, "Models, algorithms and applications to the mission planning system of imaging satellites," Systems Engineering-Theory \& Practice, vol. 31, no. 3, pp. 411-422, 2011.

[46] L. Yunfeng and W. Xiaoyue, "Composite-priority-based satellite data transmission scheduling algorithm," Journal of System Engineering, vol. 22, no. 6, pp. 644-648, 2007.

[47] L. Wei, Research on Earth Observation Satellite Mission Planning Model and Algorithm, Graduate University of Chinese Academy of Sciences (Space Science and Applied Research Center), 2008.

[48] L. Jung-Hyun, W. S. Myung, D. Chung et al., "Multi-satellite control system architecture and mission scheduling optimization," in 2012 IEEE aerospace conference, Big Sky, MT, USA, 2012.

[49] F. Zhang, J. Wang, J. Li, and N. Jing, "Multi-criteria optimal imaging scheduling based on time-ordered acyclic directed graphs," Journal of National University of Defense Technology, vol. 6, pp. 61-66, 2005.

[50] J. Wang, N. Jing, J. Li, and Z. H. Chen, "A multi-objective imaging scheduling approach for earth observing satellites," in Proceedings of the 9th annual conference on Genetic and evolutionary computation, London, England, UK, 2007.

[51] T. Schetter, M. Campbell, and D. Surka, "Multiple agent-based autonomy for satellite constellations," Artificial Intelligence, vol. 145, no. 1-2, pp. 147-180, 2003.

[52] Z. Zhang, J. Li, Y. Chen, and Y. Tan, "A research on modeling of the autonomous planning system for spacecraft," in Proceedings Of The 11Th Joint International Computer Conference: JICC 2005, Hunan, Changsha, China, 2005.

[53] H. Liu, Z. Chen, H. Chen, and J. Li, "Research on missiondriven planning method for multi-load earth observation satellites," in Proceedings of the 6th National Conference on Signal and Intelligent Information Processing and Applications: (Publishing House of Computer Engineering and Applications), pp. 163-167, Zhangiajie, Hunan Province, China, 2012.

[54] Y. Weiyi and L. Xiaolu, "Multi-satellite autonomous collaboration and mission planning based on dynamic contract network," in Proceedings of the 6th Annual Conference on High Resolution Earth Observation (Part 1), pp. 231-258, Chengdu, Sichuan Province, China, 2019.

[55] L. Shouyi, Research and Application of Agent-Based Modeling and Simulation Methods for Complex Systems, National University of Defense Technology, 2005.

[56] L. Hao, Research on Application of Modeling and Simulation of Autonomous Multi-Satellite System Based on Agent, Na-tional University of Defense Technology, 2007.

[57] Z. Xiaoguang, Research on Key Technologies of Multi-Agentbased Space Autonomous Operation System, Graduate University of Chinese Academy of Sciences (Space Science and Applied Research Center), 2007.

[58] J. M. Wang, J. F. Li, and Y. J. Tan, "Study on heuristic algorithm for dynamic scheduling problem of earth observing satellites," in Eighth Acis International Conference on Software Engineering, Qingdao, Shandong Province, China, 2007.

[59] P. Han, Z. He, Y. Geng, Y. Guo, C. Li, and G. Zhao, "Mission planning for agile earth observing satellite based on genetic algorithm," in 2019 Chinese Control Conference (CCC), pp. 2118-2123, Guangzhou, China, 2019.

[60] J. Wei, H. Huicheng, and L. Yijun, "A review of research on earth observation satellite mission planning," Systems Engi-neering and Electronics, vol. 35, no. 9, pp. 18781885, 2013.

[61] W. Farui, Research on Autonomous Mission Planning Method of Microsatellite Based on Improved Genetic Algorithm, Harbin Institute of Technology, 2017.

[62] Y. J. Song, P. Wang, Z. S. Zhang, L. N. Xing, and Y. W. Chen, "Improved genetic algorithm for multi-satellite mission planning," Control Theory and Applications, vol. 36, no. 9, pp. 1391-1397, 2019.

[63] J. Kennedy, Swarm Intelligence, Elsevier, 2001.

[64] Y. Li, S. Wang, Q. Chen, and X. Wang, "Comparative study of several new swarm intelligence optimization algorithms," Computer Engineering and Applications, vol. 56, no. 22, pp. 1-12, 2020.

[65] A. Colorni, M. Dorigo, and V. Maniezzo, "Distributed optimization by ant colonies," in Proceedings of the first European conference on artificial life, Italy, 1991.

[66] B. C. Bai, R. J. He, J. F. Li, and Y. W. Chen, "Satellite singleorbit mission synthesis observation problem and its dynamic programming algorithm," Systems Engineering and Electronics, vol. 31, no. 7, pp. 1738-1742, 2009. 
[67] C. Derong, Research on Multi-Satellite Joint Imaging Mission Planning Based on Ant Colony Algorithm, University of Electronic Science and Technology of China, 2012.

[68] C. Yuning, X. Lining, and C. Yingwu, "Smart satellite scheduling based on ant colony algorithm," Science Technology and Engineering, vol. 11, no. 3, pp. 484-489, 2011.

[69] M. G. Hinchey, R. Sterritt, and C. Rouff, "Swarms and swarm intelligence," Computer, vol. 40, no. 4, pp. 111-113, 2007.

[70] J. Kennedy and R. Eberhart, "Particle swarm optimization," in Proceedings of ICNN'95-international conference on neural networks, Perth, WA, Australia, 2002.

[71] H. Wei and Z. Xueqing, "A multi-satellite mission planning algorithm based on discrete particle swarm," Radio Engineering, vol. 45, no. 1, 2015.

[72] H. Chen, "Approach for earth observation satellite real-time and playback data transmission scheduling," Journal of Systems Engineering and Electronics, vol. 26, no. 5, pp. 982-992, 2015.

[73] F. Glover, "Tabu search-part I," ORSA Journal on computing, vol. 1, no. 1, pp. 89-98, 1989.

[74] F. Glover, "Tabu search-part II," ORSA Journal on computing, vol. 2, no. 1, 2017.

[75] Y. W. Chen, Y. S. Fang, J. F. Li, and H. E. RJ, “Constraint programming model for satellite task scheduling problem," Journal of National University of Defense Technology, vol. 5, pp. 126-132, 2006.

[76] I. Blöchliger and N. Zufferey, "A graph coloring heuristic using partial solutions and a reactive tabu scheme," Computers \& Operations Research, vol. 35, no. 3, pp. 960-975, 2008.

[77] M. Steinbrunn, G. Moerkotte, and A. Kemper, "Heuristic and randomized optimization for the join ordering problem," VLDB Journal, vol. 6, no. 3, pp. 191-208, 1997.

[78] Z. J. Huan and W. Huilin, "Research on electronic reconnaissance satellite Mission planning based on simulated annealing algorithm," Journal of the Academy of Equipment Command \& Technology, vol. 21, no. 3, pp. 62-66, 2010.

[79] Z. Chao and L. Yanbin, "Multi-agile satellite coordinated task planning and scheduling method," Science Technology and Engineering, vol. 17, no. 22, pp. 271-277, 2017.

[80] A. H. Land and A. G. Doig, An Automatic Method for Solving Discrete Programming Problems, Springer, Berlin Heidelberg, 2010.

[81] C. G. Valicka, D. Garcia, A. Staid et al., "Mixed-integer programming models for optimal constellation scheduling given cloud cover uncertainty," European Journal of Operational Research, vol. 275, no. 2, pp. 431-445, 2019.

[82] C. Xiaogeng, Research on Agile Autonomous Satellite Scheduling Algorithm, National University of Defense Technology, 2017.

[83] L. Song, B. Guoqing, and C. Yingwu, "A method for predicting the scheduling of imaging tasks in the earth observation network," Journal of Astronautics, vol. 36, no. 5, pp. 583-588, 2015.

[84] C. Li and Y. Chen, "Data-driven onboard scheduling for an autonomous observation satellite," in 27th International Joint Conference on Artificial Intelligence (ijcai), The Swedish, 2018.

[85] W. Haijiao, Research on Satellite Scale Online Scheduling Method Based on Reinforcement Learning, University of Chinese Academy of sciences (National Center for space science, Chinese Academy of Sciences), 2018.
[86] Y. Min and Z. Min, "Design of Autonomous Scheduling of small satellite tasks based on fuzzy neural network," Journal of Astronautics, vol. 28, no. 2, pp. 385-388, 2007.

[87] H. Wang, Z. Yang, W. Zhou, and D. Li, "Online scheduling of image satellites based on neural networks and deep reinforcement learning," Chinese Journal of Aeronautics, vol. 32, no. 4, pp. 1011-1019, 2019.

[88] Y. Dong, Z. Zhang, X. Li, W. Li, and G. Zhang, "Research progress and application analysis of distributed space systems," Unmanned System Technology, vol. 3, no. 5, pp. 13-20, 2020. 